\title{
Stochastic Optimal Robin Boundary Control Problems of Advection-Dominated Elliptic Equations
}

\author{
Peng Chen 1 . Alfio Quarteroni ${ }^{1}{ }^{2} \cdot$ Gianluigi Rozza $^{1}$
}

\begin{abstract}
In this work we deal with a stochastic optimal Robin boundary control problem constrained by an advection-dffusion-reaction elliptic equation with advection-dominated term. We assume that the uncertainty comes from the advection filed and consider a stochastic Robin boundary condition as control function. A stochastic saddle point system is formulated and proved to be equivalent to the first order optimality system for the optimal control problem, based on which we provide the existence and uniqueness of the optimal solution as well as some results on stochastic regularity with respect to the random variables. Stabilized finite element approximations in physical space and collocation approximations in stochastic space are applied to discretize the optimality system. A global error estimate in the product of physical space and stochastic space for the numerical approximation is derived. Illustrative numerical experiments are provided.
\end{abstract}

Keywords. stochastic optimal Robin boundary control, saddle point formulation, stochastic regularity, finite element stabilization, stochastic collocation approximation, error estimate

\section{Introduction}

Design and optimization of physical and engineering systems can be formulated as optimal control problems. The latter usually aim at the determination of the forces or boundary conditions in a system of partial differential equations, through the minimization of suitable objective or cost functionals. Deterministic optimal control problems constrained by partial differential equations have been well developed and investigated for several decades (see, e.g., [21, 14, 29]), while the development of stochastic optimal control problem constrained by stochastic partial differential equations can still be considered to be in its infancy, see the very recent work of [18, 15, 26]. In [18, stochastic optimal control problem constrained by stochastic steady diffusion problem with deterministic distributed control function is introduced and an error estimate for the Galerkin approximation of the optimality system in both physical space and stochastic space is provided. The work [15] deals with deterministic Neumann boundary control with error estimate for the same numerical approximation based on stochastic steady diffusion problem. The existence of a local optimal solution has also been demonstrated. However, the global existence as well as uniqueness of the optimal solution remain to be investigated. In [26], numerical experiments are conducted with 'pure' stochastic control function as well as 'semi' stochastic control function for an optimal control problem constrained by stochastic steady diffusion problem.

\footnotetext{
${ }^{1}$ Modelling and Scientific Computing, CMCS, Mathematics Institute of Computational Science and Engineering, MATHICSE, Ecole Polytechnique Fédérale de Lausanne, EPFL, Station 8, CH-1015 Lausanne, Switzerland. Peng Chen (peng.chen@epfl.ch), Alfio Quarteroni (alfio.quarteroni@epfl.ch), Gianluigi Rozza (gianluigi.rozza@epfl.ch)

${ }^{2}$ Modellistica e Calcolo Scientifico, MOX, Dipartimento di Matematica F. Brioschi, Politecnico di Milano, P.za Leonardo da Vinci 32, I-20133, Milano, Italy
} 
In this work, a stochastic Robin optimal control problem constrained by an advection-diffusionreaction equation with advection-dominated term is studied. In order to analyze the existence and uniqueness of the optimal solution as well as the convergence of numerical approximation, saddle point formulation for linear-quadratic type of optimal control problem in the deterministic case has been developed and fully analyzed [5, 8. We take advantage of this formulation in the stochastic Robin optimal control problem to study the theoretical properties of the optimal solution and the numerical properties of approximation in both physical and stochastic spaces. We first derive a stochastic saddlepoint system [8, 5] and prove that it is equivalent to the first order optimality system for the stochastic Robin boundary control problem. The global existence and uniqueness of the optimal solution is obtained via Brezzi's theorem [7] for the saddle point formulation. Moreover, the optimal solution of the stochastic saddle-point system is proven to depend regularly on the random variables. Thanks to this regularity, we are able to use stochastic collocation approximation 2 for the discretization of random variables and obtain a priori error estimate of the numerical approximation. As for the discretization of the physical domain, we apply stabilized finite element approximation 25, 17, 16. and provide a priori error estimate. Based on these two approximations, a global error estimate for their combination is derived. Finally, we verify the correctness of the theoretical error estimates by numerical experiments in both low (of order $O(1)$ ) and high (of order $O(100)$ ) stochastic dimensions.

In section 2, the stochastic Robin boundary control problem constrained by a stochastic advection dominated elliptic equation is introduced. We derive the stochastic saddle point system and prove it to be equivalent to the optimality system. In the following section 3 , the stochastic regularity of the solution is obtained by applying Brezzi's theorem for saddle point system recursively. Section 4 is attributed to the stabilized finite element approximation in physical space and stochastic collocation approximation in stochastic space as well as the error estimates of these approximations, followed by section 5 with numerical experiments of the approximation. Some concluding remarks are given in the last section 6 .

\section{Stochastic Robin boundary control problem}

\subsection{Problem definition}

We denote a complete probability space by $(\Omega, \mathcal{F}, P)$, where $\Omega$ is a set of outcomes $\omega \in \Omega, \mathcal{F}$ is $\sigma$-algebra of events and $P$ assigns probabilities to the events as $P: \mathcal{F} \rightarrow(0,1)$ with $P(\Omega)=1$ 13. $D$ represents an open and bounded physical domain in $\mathbb{R}^{d}(d=2,3)$ with Lipschitz continuous boundary $\partial D$. For a stochastic function defined in $D \times \Omega$, we introduce the stochastic Hilbert space $L^{2}\left(\Omega ; L^{2}(D)\right)$, or $\mathcal{L}^{2}(D)$ by calligraphic letter for convenience [18, equipped with the norm

$$
\|v\|_{\mathcal{L}^{2}(D)}=\left(\int_{\Omega} \int_{D} v^{2} d x d P\right)^{1 / 2}<\infty,
$$

Similarly, we denote $\mathcal{L}^{2}(\partial D) \equiv L^{2}\left(\Omega ; L^{2}(\partial D)\right)$ and $\mathcal{H}^{s}(D) \equiv L^{2}\left(\Omega ; H^{s}(D)\right)$ for $s \geq 0$.

Our stochastic Robin boundary control consists in finding a stochastic Robin boundary condition $g \in \mathcal{L}^{2}(\partial D)$ in order to minimize the following quadratic cost functional

$$
\mathcal{J}(u, g):=\frac{1}{2}\left\|u-u_{d}\right\|_{\mathcal{L}^{2}(D)}^{2}+\frac{\alpha}{2}\|g\|_{\mathcal{L}^{2}(\partial D)}^{2}
$$

constrained by the stochastic elliptic problem featuring a stochastic advection-dominated term

$$
\left\{\begin{array}{l}
-\nabla \cdot(a(x) \nabla u(x, \omega))+\boldsymbol{b}(x, \omega) \cdot \nabla u(x, \omega)+c(x) u(x, \omega)=f(x) \quad \text { in } D \times \Omega, \\
a(x) \nabla u(x, \omega) \cdot \boldsymbol{n}+k(x) u(x, \omega)=g(x, \omega) \quad \text { on } \partial D \times \Omega,
\end{array}\right.
$$

where $u_{d} \in \mathcal{L}^{2}(D)$ is the observation, $\alpha>0$ is a regularization coefficient, $a, \boldsymbol{b}, c$ are the diffusion, advection and reaction coefficients, respectively, $f$ is a force term, $k$ is Robin coefficient and $\boldsymbol{n}$ is the unit outward normal direction along the boundary. We make the following assumptions for $a, b, c, k$ 
Assumption 1 The uncertainty is presented on the advection-dominated term through the random coefficient $\boldsymbol{b}: D \times \Omega \rightarrow \mathbb{R}^{d}$, which satisfies $\boldsymbol{b} \in\left(\mathcal{L}^{\infty}(\bar{D})\right)^{d}, \nabla \cdot \boldsymbol{b}(x, \omega) \in \mathcal{L}^{\infty}(D)$ and can be written as a linear function of finite random variables by, e.g., truncation of Karhunen-Loève expansion [27],

$$
\boldsymbol{b}(x, \omega)=\boldsymbol{b}_{0}(x)+\sum_{n=1}^{N} \boldsymbol{b}_{n}(x) y_{n}(\omega),
$$

where $y_{n}: \Omega \rightarrow \Gamma_{n}, n=1, \ldots, N$ are uncorrelated and have zero mean and unit variance.

Assumption 2 There exist positive constants $0<r<R<\infty$ such that the diffusion coefficient

$$
r<a(x)<R \quad \text { a.e. in } \bar{D} \text {. }
$$

As customary, a.e stands for almost everywhere, meaning everywhere except for possible set with zero measure, and $\bar{D}=D \cup \partial D$. Moreover, we assume that $c \in L^{\infty}(\bar{D}), f \in L^{2}(D), k \in L^{2}(\partial D)$ as well as the relations

$$
-\frac{1}{2} \nabla \cdot \boldsymbol{b}(x, \omega)+c(x) \geq r^{\prime}>0 \quad \text { a.e. in } D \times \Omega, \text { with } r^{\prime}<r,
$$

and

$$
k(x)+\frac{1}{2} \boldsymbol{b}(x, \omega) \cdot \boldsymbol{n}(x) \geq 0 \quad \text { a.e. on } \partial D \times \Omega .
$$

Let us introduce the bilinear form $\mathcal{B}(\cdot, \cdot): \mathcal{H}^{1}(D) \times \mathcal{H}^{1}(D) \rightarrow \mathbb{R}$, defined as

$$
\begin{aligned}
\mathcal{B}(u, v) & :=(a \nabla u, \nabla v)+(\boldsymbol{b} \cdot \nabla u, v)+(c u, v)+(k u, v)_{\partial D} \\
& \equiv \int_{\Omega} \int_{D} a \nabla u \cdot \nabla v d x d P+\int_{\Omega} \int_{D}(\boldsymbol{b} \cdot \nabla u) v d x d P+\int_{\Omega} \int_{D} c u v d x d P+\int_{\Omega} \int_{\partial D} k u v d \gamma d P,
\end{aligned}
$$

and the linear functional $\mathcal{F}(\cdot): \mathcal{H}^{1}(D) \rightarrow \mathbb{R}$ defined as

$$
\mathcal{F}(v):=(f, v)+(g, v)_{\partial D} \equiv \int_{\Omega} \int_{D} f v d x d P+\int_{\Omega} \int_{\partial D} g v d \gamma d P
$$

then the weak formulation of problem $(2.2)$ can be written as

$$
\mathcal{B}(u, v)=\mathcal{F}(v), \quad \forall v \in \mathcal{H}^{1}(D) .
$$

Theorem 2.1 Provided that all the data satisfy Assumption 1 and Assumption 2, we have that there exists a unique solution $u \in \mathcal{H}^{1}(D)$ to problem (2.2), and for a suitable constant $C$, it holds

$$
\|u\|_{\mathcal{H}^{1}(D)} \leq C\left(\|f\|_{L^{2}(D)}+\|g\|_{\mathcal{L}^{2}(\partial D)}\right) .
$$

The proof follows the same lines as in the deterministic case [25] and is omitted here for simplicity.

\subsection{Stochastic saddle point formulation}

We apply Lagrangian approach for the derivation of an optimality system to solve optimal control problem (2.1) subject to the constraint (2.9). The Lagrangian functional is defined as [29]

$$
\mathcal{L}(u, g, p)=\mathcal{J}(u, g)+\mathcal{B}(u, p)-\mathcal{F}(p),
$$

where $p$ is the Lagrangian multiplier or adjoint variable in $\mathcal{H}^{1}(D)$. 
Lemma 2.2 The first-order necessary optimality conditions of the Robin boundary control problem is equivalent to the following stochastic optimality system: to find $u \in \mathcal{H}^{1}(D), p \in \mathcal{H}^{1}(D), g \in \mathcal{L}^{2}(\partial D)$,

$$
\text { s.t. }\left\{\begin{aligned}
\mathcal{B}(u, \tilde{u}) & =\mathcal{F}(\tilde{u}) \quad \forall \tilde{u} \in \mathcal{H}^{1}(D), \\
\mathcal{B}^{\prime}(p, \tilde{p}) & =\left(u_{d}-u, \tilde{p}\right) \quad \forall \tilde{p} \in \mathcal{H}^{1}(D), \\
\alpha(g, \tilde{g})_{\partial D} & =(p, \tilde{g})_{\partial D} \quad \forall \tilde{g} \in \mathcal{L}^{2}(\partial D),
\end{aligned}\right.
$$

where $\mathcal{B}^{\prime}(p, \tilde{p})=\mathcal{B}(\tilde{p}, p)$ is the adjoint bilinear form.

The stochastic optimality system 2.12 is obtained by taking Gâteaux or directional derivative of the Lagrangian functional with respective to the variables $p, u$ and $g$ respectively, and setting them to be zero, which employs the same procedure as in the deterministic case, see [20] for instance. This optimality system has also been studied in [18, 15, 26], with only local existence of the optimal solution obtained. In the following, we derive a stochastic saddle point formulation of the optimal control problem (2.1) and demonstrate the global existence and uniqueness of the optimal solution.

First of all, let us introduce new variables $\underline{u}=(u, g) \in \mathcal{U}$ and $\underline{v}=(v, h) \in \mathcal{U}$, where the stochastic tensor product space $\mathcal{U}=\mathcal{H}^{1}(D) \times \mathcal{L}^{2}(\partial D)$ is equipped with the norm $\|\underline{u}\|_{\mathcal{U}}=\|u\|_{\mathcal{H}^{1}(D)}+\|g\|_{\mathcal{L}^{2}(\partial D)}$. Define a bilinear form $\mathcal{A}(\cdot, \cdot): \mathcal{U} \times \mathcal{U} \rightarrow \mathbb{R}$

$$
\mathcal{A}(\underline{u}, \underline{v}):=(u, v)+\alpha(g, h)_{\partial D} \quad \forall \underline{u}, \underline{v} \in \mathcal{U},
$$

which is related to the cost functional 2.1 as follows,

$$
\mathcal{J}(u, g)=\frac{1}{2} \mathcal{A}(\underline{u}, \underline{u})-\left(u_{d}, u\right)+\frac{1}{2}\left(u_{d}, u_{d}\right) .
$$

Write $\underline{u}_{d}=\left(u_{d}, 0\right) \in \mathcal{U}$ as the new observation variable, we have the equivalence $\left(\underline{u}_{d}, \underline{u}\right)=\left(u_{d}, u\right)$, so that minimizing the cost functional (2.1) is not different, up to a constant $\left(u_{d}, u_{d}\right) / 2$, than minimizing the following cost functional (still denoted by $\mathcal{J}$ )

$$
\mathcal{J}(\underline{u}):=\frac{1}{2} \mathcal{A}(\underline{u}, \underline{u})-\left(\underline{u}_{d}, \underline{u}\right) .
$$

Furthermore, introduce the affine form by slight abuse of notation $\mathcal{B}(\cdot, \cdot): \mathcal{U} \times \mathcal{H}^{1}(D) \rightarrow \mathbb{R}$

$$
\mathcal{B}(\underline{u}, q):=\mathcal{B}(u, q)-(g, q)_{\partial D}, \quad \forall \underline{u} \in \mathcal{U}, \forall q \in \mathcal{H}^{1}(D) .
$$

By this new definition, we have the following minimization problem equivalent to the original one of minimizing the cost functional 2.1) subject to the stochastic constraint (2.9), which is

$$
\left\{\begin{array}{c}
\min _{\underline{u} \in \mathcal{U}_{a d}} \mathcal{J}(\underline{u})=\frac{1}{2} \mathcal{A}(\underline{u}, \underline{u})-\left(\underline{u}_{d}, \underline{u}\right), \\
\text { s.t. } \mathcal{B}(\underline{u}, q)=(f, q), \quad \forall q \in \mathcal{H}^{1}(D) .
\end{array}\right.
$$

Moreover, the equivalence between minimization problem (2.17) and the saddle point problem

$$
\text { to find }(\underline{u}, p) \in \mathcal{U} \times \mathcal{H}^{1}(D), \text { s.t. } \quad\left\{\begin{aligned}
\mathcal{A}(\underline{u}, \underline{v})+\mathcal{B}(\underline{v}, p) & =\left(\underline{u}_{d}, \underline{v}\right), \quad \forall \underline{v} \in \mathcal{U}, \\
\mathcal{B}(\underline{u}, q) & =(f, q), \quad \forall q \in \mathcal{H}^{1}(D) .
\end{aligned}\right.
$$

is established by the following proposition extended from deterministic case to the stochastic case

Proposition 2.3 [5, 8] Assume that the bilinear form $\mathcal{A}$ is symmetric, continuous, non-negative and strongly coercive on the kernel space $\mathcal{U}_{0}:=\left\{\exists \underline{u} \in \mathcal{U}: \mathcal{B}(\underline{u}, q)=0 \quad \forall q \in \mathcal{H}^{1}(D)\right\}$. Assume also that the bilinear form $\mathcal{B}$ is continuous and satisfies the compatibility condition (inf-sup condition) 2.22). 
Then the minimization problem (2.17) and the saddle point formulation (2.18) are equivalent.

From the above proposition, we immediately obtain the following lemma:

Lemma 2.4 The minimization problem 2.1) subject to the stochastic problem 2.9 is equivalent to the saddle point problem 2.18).

Proof We only need to verify the assumptions in Proposition 2.3. By definition (2.13), we have $\mathcal{A}(\underline{u}, \underline{v})=\mathcal{A}(\underline{u}, \underline{v})$ and $\mathcal{A}(\underline{u}, \underline{u}) \geq 0$ so that $\mathcal{A}$ is symmetric and non-negative. The continuity of $\mathcal{A}$ on $\mathcal{U} \times \mathcal{U}$ is evident with the following estimate

$$
\mathcal{A}(\underline{u}, \underline{v}) \leq\|u\|_{\mathcal{H}^{1}(D)}\|v\|_{\mathcal{H}^{1}(D)}+\alpha\|g\|_{\mathcal{L}^{2}(\partial D)}\|h\|_{\mathcal{L}^{2}(\partial D)} \leq C_{\alpha}\|\underline{u}\|_{\mathcal{U}}\|\underline{v}\|_{\mathcal{U}},
$$

where $C_{\alpha}$ is a constant depending on $\alpha$. On $\mathcal{U}_{0}$, we have $\mathcal{B}(\underline{u}, q)=0$ so that $\mathcal{B}(u, q)=(g, q)_{\partial D}, \forall q \in$ $\mathcal{H}^{1}(D)$. Hence, it holds by Lax-Milgram theorem that $\|u\|_{\mathcal{H}^{1}(D)} \leq R^{\prime} / r^{\prime}\|g\|_{\mathcal{L}^{2}(\partial D)}$. With this estimate, the coercivity of $\mathcal{A}$ follows:

$$
\mathcal{A}(\underline{u}, \underline{u})=\|u\|_{\mathcal{L}^{2}(D)}^{2}+\alpha\|g\|_{\mathcal{L}^{2}(\partial D)}^{2} \geq \frac{\alpha r^{\prime 2}}{2 R^{\prime 2}}\|u\|_{\mathcal{H}^{1}(D)}+\frac{\alpha}{2}\|g\|_{\mathcal{L}^{2}(\partial D)}^{2} \geq \frac{\alpha r^{\prime 2}}{2 R^{\prime 2}}\|\underline{u}\| \|_{\mathcal{U}}
$$

As for the continuity of the bilinear form $\mathcal{B}$ on $\mathcal{U} \times \mathcal{H}^{1}(D)$, we have by definition 2.16 that

$$
\mathcal{B}(\underline{u}, q) \leq R^{\prime}|| u\left\|_{\mathcal{H}^{1}(D)}|| q\right\|_{\mathcal{H}^{1}(D)}+\|g\|_{\mathcal{L}^{2}(\partial D)}\|q\|_{\mathcal{L}^{2}(\partial D)} \leq \max \left(R^{\prime}, 1\right)\|\underline{u}\|_{\mathcal{U}}\|q\|_{\mathcal{H}^{1}(D)} .
$$

The compatibility (inf-sup) condition of $\mathcal{B}$ on $\mathcal{U} \times \mathcal{H}^{1}(D)$ is shown by the following estimate

$$
\sup _{0 \neq \underline{v} \in \mathcal{U}} \frac{\mathcal{B}(\underline{v}, q)}{\|\underline{v}\|_{\mathcal{U}}}=\sup _{0 \neq(v, h) \in \mathcal{U}} \frac{\mathcal{B}(v, q)-(h, q)_{\partial D}}{\|v\|_{\mathcal{H}^{1}(D)}+\|h\|_{\mathcal{L}^{2}(\partial D)}} \geq \sup _{(v, 0) \in \mathcal{U}} \frac{\mathcal{B}(v, q)}{\|v\|_{\mathcal{H}^{1}(D)}} \geq r^{\prime}\|q\|_{\mathcal{H}^{1}(D)} .
$$

Thanks to the equivalence between the original minimization problem and the saddle point formulation established in Lemma 2.4 we can also obtain the global existence of a unique solution to the minimization problem, according to the following Brezzi's theorem for saddle point problem 2.18) (for the proof, see e.g. [7] or [25]).

Theorem 2.5 (Brezzi) Provided that the assumptions in Lemma 2.4 are satisfied, the saddle point problem (2.18) admits a unique solution $(\underline{u}, p) \in \mathcal{U} \times \mathcal{H}^{1}(D)$ or $(u, g, p) \in \mathcal{H}^{1}(D) \times \mathcal{L}^{2}(\partial D) \times \mathcal{H}^{1}(D)$. Further more, we have the following estimate

$$
\begin{aligned}
\|\underline{u}\|_{\mathcal{U}} & \leq \alpha_{1}\left\|u_{d}\right\|_{\mathcal{L}^{2}(D)}+\beta_{1}\|f\|_{L^{2}(D)}, \\
\|p\|_{\mathcal{H}^{1}(D)} & \leq \alpha_{2}\left\|u_{d}\right\|_{\mathcal{L}^{2}(D)}+\beta_{2}\|f\|_{L^{2}(D)} .
\end{aligned}
$$

where

$$
\alpha_{1}=\frac{2 R^{\prime 2}}{\alpha r^{\prime 2}}, \quad \beta_{1}=\frac{\alpha r^{\prime 2}+2 R^{\prime 2}}{\alpha r^{\prime 3}}, \quad \alpha_{2}=\frac{2 R^{\prime 2}+\alpha r^{\prime 2}}{\alpha r^{\prime 3}}, \quad \beta_{2}=\frac{\alpha r^{\prime 2}+2 R^{\prime 2}}{\alpha r^{\prime 4}} .
$$

Lemma 2.6 The saddle point problem 2.18) is equivalent to The optimality system 2.12).

Proof 2.18 amounts to find $(u, g, p) \in \mathcal{H}^{1}(D) \times \mathcal{L}^{2}(\partial D) \times \mathcal{H}^{1}(D)$, such that

$$
\left\{\begin{array}{c}
(u, v)+\alpha(g, h)_{\partial D}+\mathcal{B}(v, p)-(h, p)_{\partial D}=\left(u_{d}, v\right), \quad \forall v \in \mathcal{H}^{1}(D), \forall h \in \mathcal{L}^{2}(\partial D), \\
\mathcal{B}(u, q)-(g, q)_{\partial D}=(f, q), \quad \forall q \in \mathcal{H}^{1}(D) .
\end{array}\right.
$$

As we can observe, $(2.25)_{2}$ coincides with the state equation 2.12$)_{1}$. Moreover, we can recover the adjoint equation 2.12$)_{2}$ by setting $h=0$ in $2.25{ }_{1}$ (notice $\mathcal{B}(v, p)=\mathcal{B}^{\prime}(p, v)$ ) and the optimality 
condition 2.12$)_{3}$ by setting $v=0$ in $(2.25)_{1}$. Conversely, $(2.25)_{1}$ is retrieved by adding $(2.12)_{2}$ and $2.12{ }_{3}$.

Remark 2.1 Lemma 2.4 and 2.6 establish the equivalence between the minimization problem 2.1) subject to the stochastic problem (2.9), the saddle point problem (2.18), and the optimality system (2.12). In particular, the optimality system also admits a unique global optimal solution 2.1) according to Theorem 2.5. Moreover, other properties for the saddle point problem are also shared by the optimality system, in particular, the regularity properties of the optimal solution.

\section{Stochastic regularity}

The convergence properties of the numerical approximation to the stochastic optimality system (2.12) (or to the stochastic saddle point problem (2.18)) in the stochastic space are determined by the regularity of the stochastic solution $(u, g, p)$ or $(\underline{u}, p)$ and the choice of the approximation scheme. Since 2.12 is equivalent to 2.18 by Lemma 2.6 , we only need to study the regularity of the stochastic solution to the latter with respect to the random variables $y=\left(y_{1}, y_{2}, \ldots, y_{N}\right) \in \Gamma$. Our results are provided in Theorem 3.1. whose proof is based on recursively applying Brezzi's Theorem 2.5 in high dimensional stochastic space, adopting similar approach as in [10. An analytical extension of the solution to a certain complex domain is obtained as a consequence to this theorem in Corollary 3.2, whose proof follows using Taylor expansion and Newton binomial formula together with several elementary inequalities extended in high dimensional stochastic space.

Theorem 3.1 Holding the assumptions in Theorem 2.1 and Theorem 2.5, we can estimate the partial derivative of the solution to the stochastic saddle point problem (2.18) with respect to the variables $y=\left(y_{1}, \ldots, y_{N}\right)$ as follows: for all $\nu=\left(\nu_{1}, \ldots, \nu_{N}\right) \in \mathbb{N}^{N}$,

$$
\left\|\partial_{y}^{\nu} \underline{u}(y)\right\|_{U} \leq \sum_{0 \preceq \mu \preceq \nu} C_{\nu-\mu}^{\underline{u}, u_{d}}|\nu-\mu| !|| \boldsymbol{b}\left\|_{\left(L^{\infty}(D)\right)^{d}}^{\nu-\mu}\right\| \partial_{y}^{\mu} u_{d}(y)\left\|_{L^{2}(D)}+C C_{\bar{\nu}}^{u}, f|\nu| !|| \boldsymbol{b}\right\|_{\left(L^{\infty}(D)\right)^{d}}^{\nu}\|f\|_{L^{2}(D)},
$$

while for the adjoint variable we obtain the estimate

$$
\left\|\partial_{y}^{\nu} p(y)\right\|_{H^{1}(D)} \leq \sum_{0 \preceq \mu \preceq \nu} C_{\nu-\mu}^{p, u_{d}}|\nu-\mu| !|| \boldsymbol{b}\left\|_{\left(L^{\infty}(D)\right)^{d}}^{\nu-\mu}\right\| \partial_{y}^{\mu} u_{d}(y)\left\|_{L^{2}(D)}+C_{\nu}^{p, f}|\nu| !|| \boldsymbol{b}\right\|_{\left(L^{\infty}(D)\right)^{d}}^{\nu}\|f\|_{L^{2}(D)} .
$$

Here, $\mu \preceq \nu$ means that $\mu_{n} \leq \nu_{n}, \forall n=1,2, \ldots, N$, and the constant $C_{\nu-\mu}^{\underline{u}, u_{d}}=C_{\nu-\mu}^{\underline{u}, u_{d}}\left(\alpha_{1}, \alpha_{2}, \beta_{1}, \beta_{2}\right)$ is the sum of $2^{|\nu-\mu|}$ basic elements in the form of $\alpha_{1}^{n_{1}} \alpha_{2}^{n_{2}} \beta_{1}^{m_{1}} \beta_{2}^{m_{2}}$ such that $n_{1}+n_{2}+m_{1}+m_{2}=|\nu-\mu|+1$. The meaning holds the same for the other constants $C_{\nu-\mu}^{p, u_{d}}, C_{\nu}^{u}, f, C_{\nu}^{p, f}$ as coefficients for different terms.

Proof First of all, let us introduce the following point-wise saddle point formulation corresponding to 2.18 as follows: to find $(\underline{u}(y), p(y)) \in U \times H^{1}(D)$ with $U=H^{1}(D) \times L^{2}(\partial D)$, such that

$$
\left\{\begin{aligned}
\mathcal{A}(\underline{u}(y), \underline{v})+\mathcal{B}(\underline{v}, p(y)) & =\left(\underline{u}_{d}(y), \underline{v}\right), \quad \forall \underline{v} \in U, \\
\mathcal{B}(\underline{u}(y), q) & =(f, q), \quad \forall q \in H^{1}(D),
\end{aligned}\right.
$$

where we still denote $\mathcal{A}$ and $\mathcal{B}$ as the point-wise bilinear forms by slight abuse of notation. The properties of continuity for $\mathcal{A}$ and $\mathcal{B}$, coercivity for $\mathcal{A}$ and compatibility condition for $\mathcal{B}$ hold the same as in Lemma 2.4 Moreover, Brezzi's theorem verifies with the same parameters for the stability results 2.23). Explicitly, we have the point-wise stability for $y \in \Gamma$

$$
\|\underline{u}(y)\|_{U} \leq \alpha_{1}\left\|u_{d}(y)\right\|_{L^{2}(D)}+\beta_{1}\|f\|_{L^{2}(D)},
$$


and

$$
\|p(y)\|_{H^{1}(D)} \leq \alpha_{2}\left\|u_{d}(y)\right\|_{L^{2}(D)}+\beta_{2}\|f\|_{L^{2}(D)} .
$$

For $|\nu|=\nu_{1}+\nu_{2}+\cdots+\nu_{N}=0$, we obtain the estimate in the above stability results (3.4) and (3.5). For $|\nu| \geq 1$, by taking partial derivative of the point-wise saddle point problem (3.3) with respect to the random vector $y$ up to order $\nu$, we claim that the general recursive equation is given by

$$
\left\{\begin{array}{c}
\mathcal{A}\left(\partial_{y}^{\nu} \underline{u}(y), \underline{v}\right)+\mathcal{B}\left(\underline{v}, \partial_{y}^{\nu} p(y)\right)=\left(\partial_{y}^{\nu} \underline{u}_{d}(y), \underline{v}\right)-\sum_{j: \nu_{j} \neq 0} \nu_{j}\left(\boldsymbol{b}_{j} \cdot \nabla v, \partial_{y}^{\nu-e_{j}} p(y)\right), \quad \forall \underline{v} \in U, \\
\mathcal{B}\left(\partial_{y}^{\nu} \underline{u}(y), q\right)=-\sum_{j: \nu_{j} \neq 0} \nu_{j}\left(\boldsymbol{b}_{j} \cdot \nabla \partial_{y}^{\nu-e_{j}} u(y), q\right), \quad \forall q \in H^{1}(D),
\end{array}\right.
$$

where $\boldsymbol{b}_{j}, j=1,2, \ldots, N$ is the $j$-th basis in the linear expansion 2.3) and $e_{j}=(0, \cdots, 1, \cdots, 0)$ is an unit vector with the $j$-th element set to 1 and all the others to 0.

Indeed, if we suppose that $|\tilde{\nu}|=|\nu|-1$ and $|\tilde{\nu}|$ takes the form as $\nu-e_{k}$ for some $k$, by hypothesis, (3.6) holds for $\tilde{\nu}$, then we verify that it also holds for $\nu$. Taking the derivative of (3.6) with respect to $y_{k}$ and replace $\nu$ by $\nu-e_{k}$, we have

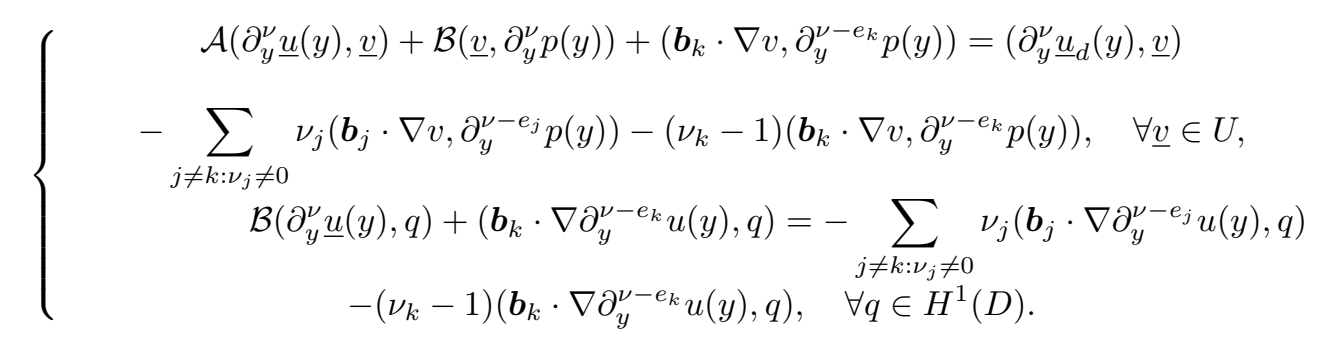

By cancelling the same terms in both sides, we retrieve the recursive equation (3.6). Applying Brezzi's theorem to the recursive equation (3.6), we have that there exist unique partial derivatives of the stochastic functions $\partial_{y}^{\nu} \underline{u}$ and $\partial_{y}^{\nu} p$ such that

$$
\begin{aligned}
\left\|\partial_{y}^{\nu} \underline{u}(y)\right\|_{U} \leq & \alpha_{1}\left(\left\|\partial_{y}^{\nu} u_{d}(y)\right\|_{L^{2}(D)}+\sum_{j: \nu_{j} \neq 0} \nu_{j}\left\|\boldsymbol{b}_{j}\right\|_{\left(L^{\infty}(D)\right)^{d}}\left\|\partial_{y}^{\nu-e_{j}} p(y)\right\|_{L^{2}(D)}\right) \\
& +\beta_{1} \sum_{j: \nu_{j} \neq 0} \nu_{j}\left\|\boldsymbol{b}_{j}\right\|_{\left(L^{\infty}(D)\right)^{d}}\left\|\partial_{y}^{\nu-e_{j}} u(y)\right\|_{L^{2}(D)}
\end{aligned}
$$

and

$$
\begin{aligned}
\left\|\partial_{y}^{\nu} p(y)\right\|_{H^{1}(D)} \leq & \alpha_{2}\left(\left\|\partial_{y}^{\nu} u_{d}(y)\right\|_{L^{2}(D)}+\sum_{j: \nu_{j} \neq 0} \nu_{j}\left\|\boldsymbol{b}_{j}\right\|_{\left(L^{\infty}(D)\right)^{d}}\left\|\partial_{y}^{\nu-e_{j}} p(y)\right\|_{L^{2}(D)}\right) \\
& +\beta_{2} \sum_{j: \nu_{j} \neq 0} \nu_{j}\left\|\boldsymbol{b}_{j}\right\|_{\left(L^{\infty}(D)\right)^{d}}\left\|\partial_{y}^{\nu-e_{j}} u(y)\right\|_{L^{2}(D)} .
\end{aligned}
$$

When $|\nu|=1$, i.e. for some $j \in \mathbb{N}, \nu=e_{j}$, using (3.4) and (3.5), the above recursive estimates (3.8) and (3.9) become

$$
\begin{aligned}
\left\|\partial_{y}^{\nu} \underline{u}(y)\right\|_{U} & \leq \alpha_{1}\left\|\partial_{y}^{\nu} u_{d}(y)\right\|_{L^{2}(D)}+\left(\alpha_{1} \alpha_{2}+\alpha_{1} \beta_{1}\right)|\nu| !|| \boldsymbol{b}\left\|_{\left(L^{\infty}(D)\right)^{d}}^{\nu}\right\| u_{d}(y) \|_{L^{2}(D)} \\
& +\left(\alpha_{1} \beta_{2}+\beta_{1} \beta_{1}\right)|\nu| !|| \boldsymbol{b}\left\|_{\left(L^{\infty}(D)\right)^{d}}^{\nu}|| f\right\|_{L^{2}(D)},
\end{aligned}
$$


and

$$
\begin{aligned}
\left\|\partial_{y}^{\nu} p(y)\right\|_{H^{1}(D)} & \leq \alpha_{2}\left\|\partial_{y}^{\nu} u_{d}(y)\right\|_{L^{2}(D)}+\left(\alpha_{2} \alpha_{2}+\alpha_{1} \beta_{2}\right)|\nu| !|| \boldsymbol{b}\left\|_{\left(L^{\infty}(D)\right)^{d}}^{\nu}\right\| u_{d}(y) \|_{L^{2}(D)} \\
& +\left(\alpha_{2} \beta_{2}+\beta_{1} \beta_{2}\right)|\nu| !|| \boldsymbol{b}\left\|_{\left(L^{\infty}(D)\right)^{d}}^{\nu}\right\| f \|_{L^{2}(D)},
\end{aligned}
$$

where $\|\boldsymbol{b}\|_{\left(L^{\infty}(D)\right)^{d}}^{\nu}=\Pi_{n=1}^{N}\left\|\boldsymbol{b}_{n}\right\|_{\left(L^{\infty}(D)\right)^{d}}^{\nu_{n}}$. For a general $\nu$ such that $|\nu| \geq 2$, we claim that the estimates (3.1) and (3.2) hold. Note that $\left\|\partial_{y}^{\nu} u(y)\right\|_{L^{2}(D)} \leq\left\|\partial_{y}^{\nu} \underline{u}(y)\right\|_{U},\left\|\partial_{y}^{\nu} p(y)\right\|_{L^{2}(D)} \leq\left\|\partial_{y}^{\nu} p(y)\right\|_{H^{1}(D)}$, by substituting (3.1) and (3.2) into the recursive formulae (3.8) with $\nu$ replaced by $\nu-e_{j}$, we have

$\left\|\partial_{y}^{\nu} \underline{u}(y)\right\|_{U} \leq \alpha_{1}\left\|\partial_{y}^{\nu} u_{d}(y)\right\|_{L^{2}(D)}$

$$
\begin{aligned}
& +\alpha_{1} \sum_{j: \nu_{j} \neq 0} \nu_{j}\left\|\boldsymbol{b}_{j}\right\|_{\left(L^{\infty}(D)\right)^{d}}\left(\sum_{0 \preceq \mu \preceq \nu-e_{j}} C_{\nu-e_{j}-\mu}^{p, u_{d}}(|\nu-\mu|-1) !|| \boldsymbol{b}\left\|_{\left(L^{\infty}(D)\right)^{d}}^{\nu-e_{j}-\mu}\right\| \partial_{y}^{\mu} u_{d}(y) \|_{L^{2}(D)}\right) \\
& +\alpha_{1} \sum_{j: \nu_{j} \neq 0} \nu_{j}\left\|\boldsymbol{b}_{j}\right\|_{\left(L^{\infty}(D)\right)^{d}}\left(C_{\nu-e_{j}}^{p, f}(|\nu|-1) ! \mid\|\boldsymbol{b}\|_{\left(L^{\infty}(D)\right)^{d}}^{\nu-e_{j}}\|f\|_{L^{2}(D)}\right) \\
& +\beta_{1} \sum_{j: \nu_{j} \neq 0} \nu_{j}\left\|\boldsymbol{b}_{j}\right\|_{\left(L^{\infty}(D)\right)^{d}}\left(\sum_{0 \preceq \mu \preceq \nu-e_{j}} C_{\nu-e_{j}-\mu}^{\underline{u}, u_{d}}(|\nu-\mu|-1) !\|\boldsymbol{b}\|_{\left(L^{\infty}(D)\right)^{d}}^{\nu-e_{j}-\mu}\left\|\partial_{y}^{\mu} u_{d}(y)\right\|_{L^{2}(D)}\right) \\
& +\beta_{1} \sum_{j: \nu_{j} \neq 0} \nu_{j}\left\|\boldsymbol{b}_{j}\right\|_{\left(L^{\infty}(D)\right)^{d}}\left(C_{\nu-e_{j}}^{\underline{u}, f}(|\nu|-1) !|| \boldsymbol{b}\left\|_{\left(L^{\infty}(D)\right)^{d}}^{\nu-e_{j}}\right\| f \|_{L^{2}(D)}\right) \\
& =\alpha_{1}\left\|\partial_{y}^{\nu} u_{d}(y)\right\|_{L^{2}(D)}+\sum_{0 \preceq \mu \preceq \nu-e_{j}}\left(\alpha_{1} C_{\nu-e_{j}-\mu}^{p, u_{d}}+\beta_{1} C_{\nu-e_{j}-\mu}^{\underline{u}, u_{d}}\right) \times \\
& \left(\sum_{j: \nu_{j} \neq 0} \nu_{j}\right)(|\nu-\mu|-1) !|| \boldsymbol{b}\left\|_{\left(L^{\infty}(D)\right)^{d}}^{\nu-\mu}\right\| \partial_{y}^{\mu} u_{d}(y) \|_{L^{2}(D)} \\
& +\left(\alpha_{1} C_{\nu-e_{j}}^{\underline{u}, f}+\beta_{1} C_{\nu-e_{j}}^{p, f}\right)\left(\sum_{j: \nu_{j} \neq 0} \nu_{j}\right)(|\nu|-1) !\|\boldsymbol{b}\|_{\left(L^{\infty}(D)\right)^{d}}^{\nu}\|f\|_{L^{2}(D)} \\
& =\sum_{0 \preceq \mu \preceq \nu} C_{\nu-\mu}^{\underline{u}, u_{d}}|\nu-\mu| !|| \boldsymbol{b}\left\|_{\left(L^{\infty}(D)\right)^{d}}^{\nu-\mu}\right\| \partial_{y}^{\mu} u_{d}(y)\left\|_{L^{2}(D)}+C_{\frac{u}{\nu}, f}^{u}|\nu| !|| \boldsymbol{b}\right\|_{\left(L^{\infty}(D)\right)^{d}}^{\nu}\|f\|_{L^{2}(D)},
\end{aligned}
$$

where the new coefficients read

$$
C_{0}^{\underline{u}, u_{d}}=\alpha_{1}, \quad C_{\nu-\mu}^{\underline{u}, u_{d}}=\left(\alpha_{1} C_{\nu-e_{j}-\mu}^{p, u_{d}}+\beta_{1} C_{\nu-e_{j}-\mu}^{\underline{u}, u_{d}}\right) \frac{|\nu|}{|\nu-\mu|} \quad \forall 0 \preceq \mu \preceq \nu-e_{j}
$$

and

$$
C_{0}^{\underline{u}, f}=\beta_{1}, \quad C_{\bar{\nu}}^{\underline{u}, f}=\left(\alpha_{1} C_{\nu-e_{j}}^{p, f}+\beta_{1} C_{\nu-e_{j}}^{\underline{u}, f}\right) .
$$

Carrying out the same procedure for $\left\|\partial_{y}^{\nu} p(y)\right\|_{H^{1}(D)}$, we obtain the estimate

$$
\left\|\partial_{y}^{\nu} p(y)\right\|_{H^{1}(D)} \leq \sum_{0 \preceq \mu \preceq \nu} C_{\nu-\mu}^{p, u_{d}}|\nu-\mu| !|| \boldsymbol{b}\left\|_{\left(L^{\infty}(D)\right)^{d}}^{\nu-\mu}\right\| \partial_{y}^{\mu} u_{d}(y)\left\|_{L^{2}(D)}+C_{\nu}^{p, f}|\nu| !|| \boldsymbol{b}\right\|_{\left(L^{\infty}(D)\right)^{d}}^{\nu}\|f\|_{L^{2}(D)},
$$

where the coefficients

$$
C_{0}^{p, u_{d}}=\alpha_{2}, \quad C_{\nu-\mu}^{p, u_{d}}=\left(\alpha_{2} C_{\nu-e_{j}-\mu}^{p, u_{d}}+\beta_{2} C_{\nu-e_{j}-\mu}^{\underline{u}, u_{d}}\right) \frac{|\nu|}{|\nu-\mu|} \quad \forall 0 \preceq \mu \preceq \nu-e_{j}
$$


and

$$
C_{0}^{p, f}=\beta_{2}, \quad C_{\nu}^{p, f}=\left(\alpha_{2} C_{\nu-e_{j}}^{p, f}+\beta_{2} C_{\nu-e_{j}}^{\underline{u}, f}\right) .
$$

By the above recursive formulae, we have that the constant $C_{\nu-\mu}^{\underline{u}, u_{d}}=C_{\nu-\mu}^{\underline{u}, u_{d}}\left(\alpha_{1}, \alpha_{2}, \beta_{1}, \beta_{2}\right)$ is the sum of $2^{|\nu-\mu|}$ basic elements in the form of $\alpha_{1}^{n_{1}} \alpha_{2}^{n_{2}} \beta_{1}^{m_{1}} \beta_{2}^{m_{2}}$ such that $n_{1}+n_{2}+m_{1}+m_{2}=|\nu-\mu|+1$. The same structure holds for the constant $C_{\nu-\mu}^{p, u_{d}}, C_{\nu}^{\underline{u}, f}$ and $C_{\nu}^{p, f}$. Notice the difference that $C_{\nu-\mu}^{\underline{u}, u_{d}}$ and $C_{\nu-\mu}^{p, u_{d}}$ are modified by some constant related to $|\nu| /|\nu-\mu| \rightarrow 1$ as $|\nu| \rightarrow \infty$ for fixed $\mu$.

A direct consequence of the regularity given in Theorem 3.1 is the analyticity property of $(\underline{u}, p)$ with respect to $y \in \Gamma$, provided the following conditions are satisfied.

Corollary 3.2 Holding all the assumptions for Theorem 3.1, and the following conditions

$$
2 K \sum_{n}^{N}|| \boldsymbol{b}_{n} \|_{\left(L^{\infty}(D)\right)^{d}}\left|y_{n}-\bar{y}_{n}\right|<1,
$$

where $K=\max \left(\alpha_{1}, \alpha_{2}, \beta_{1}, \beta_{2}\right)$ and

$$
\sum_{\mu} \frac{|\mu||y-\bar{y}|^{\mu}}{\mu !}\left\|\partial_{y}^{\mu} u_{d}(\bar{y})\right\|_{L^{2}(D)}<\infty
$$

we have the existence of an analytic expansion of the stochastic solution $(\underline{u}, p)$ to the saddle point problem (3.3) around $\bar{y} \in \Gamma$. Therefore, $(\underline{u}, p)$ can be analytically extended to the set

$$
\Sigma=\left\{y \in \mathbb{R}^{N}: \exists \bar{y} \in \Gamma \text { such that } 3.18 \text { and } 3.19 \text { hold }\right\},
$$

and we define $\Sigma(\Gamma ; \tau):=\{z \in \mathbb{C}: \operatorname{dist}(z, \Gamma) \leq \tau\} \subset \Sigma$ for the largest possible vector $\tau=\left(\tau_{1}, \ldots, \tau_{N}\right)$.

Proof The Taylor expansion of $\underline{u}(y), y \in \Gamma$ around $\bar{y} \in \Gamma$ is given by

$$
\underline{u}(y)=\sum_{\nu} \frac{\partial_{y}^{\nu} \underline{u}(\bar{y})}{\nu !}(y-\bar{y})^{\nu}
$$

where $\nu !=\nu_{1} ! \cdots \nu_{N} !$. By the bound of Theorem 3.1. we have the estimate

$$
\begin{aligned}
& \left\|\sum_{\nu} \frac{\partial_{y}^{\nu} \underline{u}(\bar{y})}{\nu !}(y-\bar{y})^{\nu}\right\|_{U} \leq \sum_{\nu} \frac{|y-\bar{y}|^{\nu}}{\nu !}\left(\sum_{0 \preceq \mu \preceq \nu} C_{\nu-\mu}^{\underline{u}, u_{d}}|\nu-\mu| !\|\boldsymbol{b}\|_{\left(L^{\infty}(D)\right)^{d}}^{\nu-\mu}\left\|\partial_{y}^{\mu} u_{d}(\bar{y})\right\|_{L^{2}(D)}\right) \\
& +\sum_{\nu} \frac{|y-\bar{y}|^{\nu}}{\nu !} C_{\bar{\nu}}^{u}, f|\nu| !|| \boldsymbol{b}\left\|_{\left(L^{\infty}(D)\right)^{d}}^{\nu}|| f\right\|_{L^{2}(D)},
\end{aligned}
$$

where $|y-\bar{y}|=\left(\left|y_{1}-\bar{y}_{1}\right|, \ldots,\left|y_{N}-\bar{y}_{N}\right|\right)$. Let us consider the second term at first, for which we introduce the generalized Newton binomial formula: for any $\eta=\left(\eta_{1}, \ldots, \eta_{N}\right) \in \mathbb{R}^{N}$ and $k=0,1,2, \ldots$, we have

$$
\sum_{|\nu|=k} \frac{k !}{\nu !} \eta^{\nu}=\left(\sum_{n=1}^{N} \eta_{n}\right)^{k}
$$

By applying (3.23), the second term of 3.22 becomes 


$$
\begin{aligned}
& \sum_{\nu} \frac{|y-\bar{y}|^{\nu}}{\nu !} C_{\nu}^{u, f}|\nu| !|| \boldsymbol{b}\left\|_{\left(L^{\infty}(D)\right)^{d}}^{\nu}|| f\right\|_{L^{2}(D)} \\
= & \|f\|_{L^{2}(D)} \sum_{k=0}^{\infty} \sum_{|\nu|=k} C \frac{u}{\nu}, f \frac{|\nu| !}{\nu !}\left(\|\boldsymbol{b}\|_{\left(L^{\infty}(D)\right)^{d}}|y-\bar{y}|\right)^{\nu} \\
\leq & K\|f\|_{L^{2}(D)} \sum_{k=0}^{\infty} \sum_{|\nu|=k} \frac{|\nu| !}{\nu !}\left(2 K\|\boldsymbol{b}\|_{\left(L^{\infty}(D)\right)^{d}}|y-\bar{y}|\right)^{\nu} \\
= & K\|f\|_{L^{2}(D)} \sum_{k=0}^{\infty}\left(2 K \sum_{n}^{N}\left\|\boldsymbol{b}_{n}\right\|_{\left(L^{\infty}(D)\right)^{d}}\left|y_{n}-\bar{y}_{n}\right|\right)^{k},
\end{aligned}
$$

where $K=\max \left(\alpha_{1}, \alpha_{2}, \beta_{1}, \beta_{2}\right)$ and the inequality comes from the estimate for the coefficient $C_{\underline{\nu}}^{\underline{u}, f}$

$$
C_{\frac{u}{\nu}, f} \leq 2^{|\nu|} K^{|\nu|+1}=K(2 K)^{\nu},
$$

which is valid by definition. Therefore, the convergence condition for 3.24 is

$$
2 K \sum_{n}^{N}|| \boldsymbol{b}_{n} \|_{\left(L^{\infty}(D)\right)^{d}}\left|y_{n}-\bar{y}_{n}\right|<1
$$

As for the first term of the estimate $(3.22)$, we have

$$
\begin{aligned}
& \sum_{\nu} \frac{|y-\bar{y}|^{\nu}}{\nu !}\left(\sum_{0 \preceq \mu \preceq \nu} C_{\nu-\mu}^{\underline{u}, u_{d}}|\nu-\mu| !|| \boldsymbol{b}\left\|_{\left(L^{\infty}(D)\right)^{d}}^{\nu-\mu}\right\| \partial_{y}^{\mu} u_{d}(\bar{y}) \|_{L^{2}(D)}\right) \\
\leq & \sum_{\mu} \frac{|y-\bar{y}|^{\mu}}{\mu !}\left\|\partial_{y}^{\mu} u_{d}(\bar{y})\right\|_{L^{2}(D)}\left(\sum_{\nu \succeq \mu} C_{\nu-\mu}^{\underline{u}, u_{d}} \frac{|\nu-\mu| !}{(\nu-\mu) !}\left(\|\boldsymbol{b}\|_{\left(L^{\infty}(D)\right)^{d}}|y-\bar{y}|\right)^{\nu-\mu}\right) \\
\leq & K \sum_{\mu} \frac{|\mu||y-\bar{y}|^{\mu}}{\mu !}\left\|\partial_{y}^{\mu} u_{d}(\bar{y})\right\|_{L^{2}(D)} \sum_{k=0}^{\infty}\left(2 K \sum_{n}^{N}\left\|\boldsymbol{b}_{n}\right\|_{\left(L^{\infty}(D)\right)^{d}}\left|y_{n}-\bar{y}_{n}\right|\right)^{k},
\end{aligned}
$$

where for the first inequality we employ the equality

$$
\sum_{\nu} \sum_{0 \preceq \mu \preceq \nu} \cdot=\sum_{\mu} \sum_{\nu \succeq \mu}
$$

and the bound

$$
\frac{1}{\nu !} \leq \frac{1}{\mu !} \frac{1}{(\nu-\mu) !}
$$

For the second inequality we replace all $\nu-\mu$ by $\nu$, bound the coefficient $C_{\nu-\mu}^{\underline{u}, u_{d}}$ by

$$
C_{\nu-\mu}^{\underline{u}, u_{d}} \leq \frac{|\nu|}{|\nu-\mu|} 2^{|\nu-\mu|} K^{|\nu-\mu|+1} \leq K|\mu|(2 K)^{|\nu-\mu|}
$$

and use the result obtained for the second term (3.24). Hence, the convergence of the first term (3.27) is guaranteed by the condition (3.26) as well as the condition (3.19), which implies that $u_{d}$ is analytic around $\bar{y}$. The same procedure holds for the Taylor expansion of

$$
p(y)=\sum_{\nu} \frac{\partial_{y}^{\nu} p(\bar{y})}{\nu !}(y-\bar{y})^{\nu}
$$


with estimate in the space of $H^{1}(D)$.

\section{Approximation and error estimates}

Numerical approximation in both the physical space and the stochastic space will be studied in this section. More specifically, we apply stabilized finite element approximation in physical space in order to address the advection dominated problem [25, 11, 4, 6, 17, 16 and employ adaptive stochastic collocation approximation in stochastic space [31, 2, 22, 23, 30, 3, to deal with the computational reduction for high-dimensional stochastic problem. Numerical properties of the approximations will be analyzed in both of the physical space and the stochastic space individually, and the analysis of a global error estimate for a combined stabilized finite element - adaptive stochastic collocation approximation will also be considered.

\subsection{Finite element approximation in physical space}

Let us introduce a regular triangulation $\mathcal{T}_{h}$ of the physical domain $D \subset \mathbb{R}^{d}, d=2,3$ such that $\bar{D}=\cup_{K \in \mathcal{T}_{h}} K$ and $\operatorname{diam}(K) \leq h$. Based on this triangulation, we define a finite element space $X_{h}^{k}$

$$
X_{h}^{k}:=\left\{v_{h} \in C^{0}(\bar{D}) \mid v_{h \mid K} \in \mathbb{P}_{k} \quad \forall K \in \mathcal{T}_{h}\right\}, \quad k \geq 0,
$$

where $\mathbb{P}_{k}, k \geq 0$ is the space of polynomials of total degree less than or equal to $k$ in the variables $x_{1}, \ldots, x_{d}$. Therefore, the element $v_{h}$ in $X_{h}^{k}$ is simply a piece-wise polynomial, and we have that $X_{h}^{k} \subset H^{1}(D)$ [25. Since both the state equation and the adjoint equation are advection dominated, a proper stabilization method is needed. Let us introduce the elliptic operator for the point-wise state equation as follows: $\forall y \in \Gamma$, define

$$
L u(y):=-\nabla \cdot(a \nabla u(y))+\boldsymbol{b}(y) \cdot \nabla u(y)+c u(y),
$$

which can be separated into a symmetric part and a skew-symmetric part $L=L_{s}+L_{s s}$, defined as

$$
L_{s} u(y)=-\nabla \cdot(a \nabla u(y))+c u(y) ; \quad L_{s s} u(y)=\boldsymbol{b}(y) \cdot \nabla u(y) .
$$

Corresponding to the adjoint equation, we define the adjoint elliptic operator: $\forall y \in \Gamma$, define

$$
L^{\prime} p(y):=-\nabla \cdot(a \nabla p(y))-\boldsymbol{b}(y) \cdot \nabla p(y)+(c-\nabla \cdot \boldsymbol{b}) p(y)
$$

and we split it into a symmetric part and a skew-symmetric part, $L^{\prime}=L_{s}^{\prime}+L_{s s}^{\prime}$, we have

$$
L_{s}^{\prime} p(y)=-\nabla \cdot(a \nabla p(y))+(c-\nabla \cdot \boldsymbol{b}) u(y) ; \quad L_{s s}^{\prime} u(y)=-\boldsymbol{b}(y) \cdot \nabla p(y) .
$$

Substituting the optimality condition 2.12 into the state equation 2.2 and taking the following stabilized weak formulation for both the state equation and adjoint equation, we obtain the stabilized and reduced optimality system in finite element space $X_{h}^{k}$ as follows [25]:

$$
\left\{\begin{array}{l}
\mathcal{B}\left(u_{h}(y), v_{h}\right)+\sum_{K \in \mathcal{T}_{h}} \delta_{K}\left(L u_{h}(y), \frac{h_{K}}{|\boldsymbol{b}|}\left(L_{s s}+\theta L_{s}\right) v_{h}\right) \\
=\frac{1}{\alpha}\left(p_{h}(y), v_{h}\right)+\left(f, v_{h}\right)+\sum_{K \in \mathcal{T}_{h}} \delta_{K}\left(f, \frac{h_{K}}{|\boldsymbol{b}|}\left(L_{s s}+\theta L_{s}\right) v_{h}\right) \quad \forall v_{h} \in X_{h}^{k}, \\
\mathcal{B}^{\prime}\left(p_{h}(y), v_{h}\right)+\sum_{K \in \mathcal{T}_{h}} \delta_{K}\left(L^{\prime} p_{h}(y), \frac{h_{K}}{|\boldsymbol{b}|}\left(L_{s s}^{\prime}+\theta L_{s}^{\prime}\right) v_{h}\right) \\
=\left(u_{d}(y)-u_{h}(y), v_{h}\right)+\sum_{K \in \mathcal{T}_{h}} \delta_{K}\left(u_{d}(y)-u_{h}(y), \frac{h_{K}}{|\boldsymbol{b}|}\left(L_{s s}^{\prime}+\theta L_{s}^{\prime}\right) v_{h}\right) \quad \forall v_{h} \in X_{h}^{k},
\end{array}\right.
$$


where $|\boldsymbol{b}|$ is the modulus of $\boldsymbol{b}$ and the parameter $\delta_{K}$ is left to be chosen, for instance,

$$
\delta_{K}=\delta(P e)=\operatorname{coth}\left(P e_{K}\right)-\frac{1}{P e_{K}}, \text { where } P e_{K}:=\frac{|\boldsymbol{b}| h_{K}}{2 a_{\min }}, \quad \forall K \in \mathcal{T}_{h} .
$$

Different stabilization methods result from the choice of $\theta$. If $\theta=0$, it corresponds to SUPG stabilization (Streamline Upwind/Petrov-Galerkin); if $\theta=1$, GALS stabilization (Galerkin/LeastSquares) is obtained. For these different stabilization methods, it has been proven that if the parameter $\delta$ is small enough and the solution of the state equation $(2.2)$ is regular enough, e.g. $u(y) \in H^{k+1}(D)$, the priori error of the approximation is bounded by the estimate $\left\|u-u_{h}\right\|_{V} \leq h^{k+1 / 2}\|u\|_{H^{k+1}(D)}$, where the norm $\|\cdot\|_{V}$ is defined according to different methods. More details about the strong consistency and accuracy of these stabilization methods are provided in 25] or 24]. As for the convergence property of the optimality system, we have the following point-wise results by "Optimize-Then-Discretize" procedure for SUPG stabilization, see similar proof in [11] for distributed optimal control problem:

Lemma 4.1 Let $k, l, m \geq 1$, and suppose that $\forall y \in \Gamma$ the solution $(u(y), g(y), p(y))$ satisfies $u(y) \in$ $H^{k+1}(D), g(y) \in H^{m+1 / 2}(\partial D)$ and $p(y) \in H^{l+1}(D)$. If the stabilization parameter satisfies

$$
\delta_{K} \leq \min \left(\frac{h_{K}^{2}}{\varepsilon C_{K}^{2}}, \frac{r^{\prime}}{\|c\|_{L^{\infty}(K)}}, \frac{r^{\prime}}{\|c-\nabla \cdot \boldsymbol{b}\|_{L^{\infty}(K)}}\right), \quad \forall K \in \mathcal{T}_{h},
$$

where $\varepsilon=a_{\max } \leq R, r^{\prime}$ is the coefficient defined in 2.5) and $C_{K}$ is the constant for the inverse inequality $\left\|\nabla v_{h}\right\|_{L^{2}(K)} \leq C_{K} h_{K}^{-1}\left\|v_{h}\right\|_{L^{2}(K)}, \forall K \in \mathcal{T}_{h}$, and we take for positive constant $\tau_{1}, \tau_{2}>0$

$$
\delta_{K}=\tau_{1} \frac{h_{K}^{2}}{\varepsilon} \text { for } P e_{K} \leq 1, \text { or } \delta_{K}=\tau_{2} h_{K} \text { for } P e_{K}>1,
$$

then the error estimate for the discretized optimal solution $\left(u_{h}(y), g_{h}(y), p_{h}(y)\right), \forall y \in \Gamma$ is obtained as

$$
\begin{aligned}
& \left\|u(y)-u_{h}(y)\right\|_{V}+\left\|g(y)-g_{h}(y)\right\|_{L^{2}(\partial D)}+\left\|p(y)-p_{h}(y)\right\|_{V} \\
& \leq C\left(\left(\varepsilon^{1 / 2}+h^{1 / 2}\right)\left(h^{k}|u(y)|_{k+1}+h^{l}|p(y)|_{l+1}\right)+h^{m+1}|g(y)|_{m+1 / 2, \partial D}\right) .
\end{aligned}
$$

where the norm $\|\cdot\|_{V}$ is defined for SUPG stabilization as

$$
\|v\|_{V}^{2}=\varepsilon|v|_{1}^{2}+r^{\prime}\|v\|_{L^{2}(K)}^{2}+\sum_{K \in \mathcal{T}_{h}} \delta_{K}\|\boldsymbol{b} \cdot \nabla v\|_{L^{2}(K)}^{2},
$$

and $|v|_{k}, k \geq 1$ is the semi-norm in the Hilbert space $H^{k}(D), k \geq 1$.

Remark 4.1 Lemma 4.1 provides a convergence result for the error between the solution of the original and that of the discretized optimal control problem over the entire domain D. The constants in the estimates of the global error depend on regularity of the optimal solution $(u, g, p)$. Similar results have also been obtained recently in [4, 6, 17].

\subsection{Collocation approximation in stochastic space}

Stochastic collocation method has been developed by combining the advantage of straightforward implementation of Monte-Carlo method and the benefit of high accuracy and fast convergence of stochastic Galerkin method [31, 2. It takes the collocation nodes in multidimensional probability space as the samples and constructs the stochastic solution by interpolation of the solution obtained from a deterministic solver at all the collocation nodes. In order to further alleviate the computational cost, sparse grid proposed by [28] has been applied in both isotropic and anisotropic manner [22, 23] as well as in quasi-optimal way with respect to the cost of computation and gain of accuracy [12. Hierarchical selection of the collocation nodes has also been applied based on the abscissae of Chebyshev and 
Gaussian-Patterson quadrature rule [1]. More comparison of analytical and numerical aspects among these stochastic computational methods can be found in, for instance, [30, 3, 9]. Thanks to the nonintrusive characteristic and fast convergence of stochastic collocation method, we will adopt it for the computation of solution to the stochastic optimality system 2.12) or the saddle point system (2.18). Let us summarize the stochastic collocation method in a general formulation at first with full tensor product grid, isotropic sparse grid and anisotropic sparse grid, respectively.

\subsubsection{Full tensor product grid collocation approximation}

The basic idea behind the construction of stochastic collocation method is to interpolate a stochastic function $u(x, y)$ in multidimensional stochastic space $\Gamma$ at a set of collocation nodes $\left\{y^{j}\right\}_{j=1}^{N_{q}}$ with each $y^{j}=\left(y_{1}^{j}, \cdots, y_{N}^{j}\right), j=1, \ldots, N_{q}$ [31, 2]. Denote $\mathcal{P}_{q}(\Gamma)$ as the multidimensional polynomial space of degree $q=\left(q_{1}, \ldots, q_{N}\right)$. The multidimensional Lagrange interpolation operator $\mathcal{I}_{q}: C\left(\Gamma ; H^{1}(D)\right) \rightarrow$ $\mathcal{P}_{q}(\Gamma) \otimes H^{1}(D)$ is defined as

$$
\mathcal{I}_{q} u(x, y)=\sum_{j=1}^{N_{q}} u\left(x, y_{j}\right) l^{j}(y) \text { with Lagrange polynomials } l^{j}(y)=\prod_{n=1}^{N} l_{n}^{j}\left(y_{n}\right), \quad j=1, \ldots, N_{q}
$$

Consequently, the expectation of the solution can be computed as

$$
\mathbb{E}\left[\mathcal{I}_{q} u\right](x)=\int_{\Gamma} u(x, y) \rho(y) d y=\sum_{j=1}^{N_{q}} u\left(x, y_{j}\right) \int_{\Gamma} l^{j}(y) \rho(y) d y=\sum_{j=1}^{N_{q}} u\left(x, y_{j}\right) w^{j},
$$

where the weight $w^{j}$ is obtained from the integral

$$
w^{j}=\int_{\Gamma}\left(\prod_{n=1}^{N} l_{n}^{j}(y)\right) \rho(y) d y=\prod_{n=1}^{N} w_{n}^{j}, j=1, \ldots, N_{q} .
$$

The variance of the solution can be obtained by the orthonormal property of Lagrange polynomial

$$
\operatorname{Var}\left[\mathcal{I}_{q} u\right](x)=\mathbb{E}\left[\left(\mathcal{I}_{q} u\right)^{2}\right](x)-\left(\mathbb{E}\left[\mathcal{I}_{q} u\right](x)\right)^{2}=\sum_{j=1}^{N_{q}} u^{2}\left(x, y_{j}\right) w^{j}-\left(\sum_{j=1}^{N_{q}} u\left(x, y_{j}\right) w^{j}\right)^{2}
$$

The choice of collocation nodes is crucial as it determines the accuracy and efficiency of stochastic collocation method. An obvious choice consists of taking the full tensor product of one dimensional set. First of all, for any one dimensional function, for instance, $u\left(y_{n}\right), n=1, \ldots, N$, we have the general interpolation operator defined as

$$
\mathcal{U}^{q_{n}} u\left(y_{n}\right)=\sum_{j_{n}=1}^{N_{q_{n}}} u\left(y_{n}^{j_{n}}\right) l_{n}^{j_{n}}\left(y_{n}\right), \quad n=1, \ldots, N
$$

on a set of collocation nodes $\Theta^{n}=\left(y_{n}^{1}, \ldots, y_{n}^{N_{q_{n}}}\right)$. The tensor product Lagrange interpolation operator $\mathcal{I}_{q}$ can thus be expressed as the tensor product of the one dimensional interpolation operator

$$
\begin{aligned}
\mathcal{I}_{q} u(y) & =\left(\mathcal{U}^{q_{1}} \otimes \cdots \otimes \mathcal{U}^{q_{N}}\right) u(y) \\
& =\sum_{j_{1}=1}^{N_{q_{1}}} \cdots \sum_{j_{N}=1}^{N_{q_{N}}} u\left(y_{1}^{j_{1}}, \ldots, y_{N}^{j_{N}}\right)\left(l_{1}^{j_{1}}\left(y_{1}\right) \otimes \cdots \otimes l_{N}^{j_{N}}\left(y_{N}\right)\right) .
\end{aligned}
$$

The convergence rate of the tensor product interpolation operator in the multidimensional stochastic space $C(\Gamma ; V)$ is summarized in the following proposition, see [2] for a detailed proof. 
Proposition 4.2 Suppose that the function $u$ can be analytically extended to a complex domain $\Sigma(\Gamma ; \tau)$. The following convergence estimate holds for the multidimensional full tensor product interpolation operator $\mathcal{I}_{q}$

$$
\left\|u-\mathcal{I}_{q} u\right\|_{L^{2}(\Gamma ; V)} \leq C \sum_{n=1}^{N}\left(\frac{1}{r_{n}}\right)^{q_{n}},
$$

where the constants $r_{n}$ are defined via $\tau_{n}$ and $\Gamma_{n}$ as

$$
r_{n}=\frac{2 \tau_{n}}{\left|\Gamma_{n}\right|}+\sqrt{1+\frac{4 \tau_{n}^{2}}{\left|\Gamma_{n}\right|^{2}}}>1, \quad n=1, \ldots, N
$$

\subsubsection{Isotropic and anisotropic sparse grid collocation approximation}

The set of collocation nodes for the full tensor product interpolation operator $\mathcal{I}_{q}$ are the product of $\Theta=\Theta^{q_{1}} \times \cdots \Theta^{q_{N}}$ with $N_{q}=\prod_{n=1}^{N} N_{q_{n}}$ collocation nodes in total. When $N$ becomes large, the full tensor product interpolation becomes computationally prohibitive. In order to alleviate the "curse of dimensionality", we adopt Smolyak sparse grid [28, 31. For isotropic interpolation with the same degree $q \geq N$ for one dimensional polynomial space in each direction, the Smolyak interpolation operator is defined as

$$
\mathcal{S}_{q} u(y)=\sum_{q-N+1 \leq|i| \leq q}(-1)^{q-|i|}\left(\begin{array}{c}
N-1 \\
q-|i|
\end{array}\right)\left(\mathcal{U}^{i_{1}} \otimes \cdots \otimes \mathcal{U}^{i_{N}}\right) u(y),
$$

where $|i|=i_{1}+\cdots+i_{N}$, the multivariate index $i$ can also be defined via the index set

$$
X(q, N):=\left\{i \in N_{+}^{N}, \forall i_{n} \geq 1: \sum_{n=1}^{N} i_{n} \leq q\right\},
$$

and the set of collocation nodes for the sparse grid (see the middle of Figure 4.1) is thus collected as

$$
H(q, N)=\bigcup_{q-N+1 \leq|i| \leq q}\left(\Theta^{i_{1}} \times \cdots \times \Theta^{i_{N}}\right)
$$

Define $l=q-N$ as the collocation level, we have $q-N+1 \leq|i| \leq q \rightarrow l+1 \leq|i| \leq l+N$. An alternative expression of Smolyak interpolation is written with the definition of the differential operator $\Delta^{i_{n}}=\mathcal{U}^{i_{n}}-\mathcal{U}^{i_{n}-1}, n=1, \ldots, N$ and $\mathcal{U}^{0}=0$ as

$$
\begin{aligned}
\mathcal{S}_{q} u(y) & =\sum_{i \in X(q, N)}\left(\Delta^{i_{1}} \otimes \cdots \otimes \Delta^{i_{N}}\right) u(y) \\
& =\mathcal{S}_{q-1} u(y)+\sum_{|i|=q}\left(\Delta^{i_{1}} \otimes \cdots \otimes \Delta^{i_{N}}\right) u(y) .
\end{aligned}
$$

The above formula allows us to discretize the stochastic space in hierarchical structure based on nested collocation nodes, such as the extrema of Chebyshev polynomials or Gauss-Patterson nodes, leading to Clenshaw-Curtis cubature rule or Gauss-Patterson cubature rule, respectively [23, 19].

Smolyak sparse grid is isotropic in every one-dimensional polynomial space. However, the convergence rate of the solution in each polynomial space may vary due to different importance of each random variable, which helps to reduce the dimensionality by anisotropic sparse grid, written as

$$
\mathcal{S}_{q}^{\alpha} u(y)=\sum_{i \in X_{\alpha}(q, N)}\left(\Delta^{i_{1}} \otimes \cdots \otimes \Delta^{i_{N}}\right) u(y)
$$


with the weighted index

$$
X_{\alpha}(q, N):=\left\{i \in N_{+}^{N}, i \geq 1: \sum_{n=1}^{N} i_{n} \alpha_{n} \leq \min (\alpha) q\right\}
$$

where $\alpha=\left(\alpha_{1}, \ldots, \alpha_{N}\right)$ represents the weights in different directions, obtained either from a priori error estimation or a posteriori error estimation, see 22]. Figure 4.1 displays the full tensor product grid, the sparse grid and the anisotropic sparse grid based on Clenshaw-Curtis cubature rule. We can observe that the isotropic and anisotropic sparse grids are far coarser than the full tensor product grid, leading to considerable reduction of the high dimensional stochastic computation.
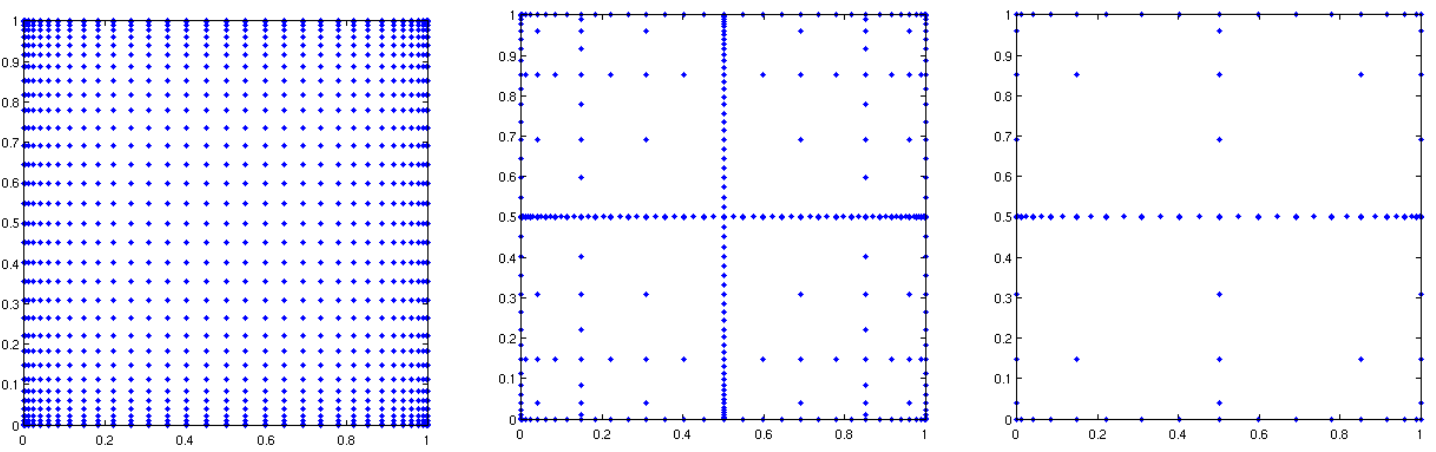

Figure 4.1: Two dimensional collocation nodes by Clenshaw-Curtis cubature rule in tensor product grid $q=8$ (Left), sparse grid $q=8$ (Middle), anisotropic sparse grid $q=8$ and $\alpha=(1,1.5)$ (Right)

The convergence analysis of the isotropic and anisotropic Smolyak sparse grids stochastic collocation methods have been studied in detail in [23] and 22]. Let us summarize them briefly in the following proposition

Proposition 4.3 Suppose that the function $u$ can be analytically extended to a complex domain $\Sigma(\Gamma ; \tau)$. Using isotropic Smolyak sparse grid with Clenshw-Curtis collocation nodes yields the following convergence estimate

$$
\left\|u-\mathcal{S}_{q} u\right\|_{L^{2}(\Gamma ; V)} \leq C N_{q}^{-r}
$$

where $C$ is a constant independent of $N_{q}$ and $r, N_{q}=\# H(q, N)$ is the number of collocation nodes, $r$ is defined as $r=\min \left(\log \left(\sqrt{r_{1}}\right), \ldots, \log \left(\sqrt{r_{N}}\right)\right) /(1+\log (2 N))$ with $r_{1}, \ldots, r_{N}$ defined in (4.19). Then using the anisotropic Smolyak sparse grid, still with Clenshw-Curtis collocation nodes, we have instead

$$
\left\|u-\mathcal{S}_{q}^{\alpha} u\right\|_{L^{2}(\Gamma ; V)} \leq C N_{q}^{-r(\alpha)}
$$

where $r(\alpha)=\min (\alpha)(\log (2) e-1 / 2) /\left(\log (2)+\sum_{n=1}^{N} \min (\alpha) / \alpha_{n}\right)$ and $\alpha_{n}=\log \left(\sqrt{r_{n}}\right), n=1, \ldots, N$.

\subsection{Convergence for approximating stochastic optimal control problem}

In this section, we provide some convergence results for the stabilized finite element approximation in the physical space and stochastic collocation approximation in the stochastic space for the optimality system 2.12, or equivalently, the saddle point system 2.18. Let us denote the fully approximated solution in both the physical space and the stochastic space as $\left(u_{h, q}, g_{h, q}, p_{h, q}\right)$. We summarize in Theorem 4.4 and Theorem 4.5 the error estimates for tensor product grid collocation approximation and sparse grid collocation approximation for stochastic Robin boundary control problem, respectively. 
Theorem 4.4 Provided that the assumptions made in Corollary 3.2. Lemma 4.1 and Proposition 4.2 are satisfied, the following global error estimate for stabilized finite element approximation in the physical space and full tensor product grid collocation approximation in the stochastic space for the stochastic optimality system (2.12) (or equivalently the saddle point system 2.18)) holds

$$
\begin{aligned}
& \left\|u-u_{h, q}\right\|_{\mathcal{V}(D)}+\left\|g-g_{h, q}\right\|_{\left.\mathcal{L}^{2}(\partial D)\right)}+\left\|p-p_{h, q}\right\|_{\mathcal{V}(D)} \\
& \leq C_{s} \sum_{n=1}^{N}\left(\frac{1}{r_{n}}\right)^{q_{n}}+C_{p}\left(\varepsilon^{1 / 2}+h^{1 / 2}\right) h^{k}\left(|u|_{\mathcal{H}^{k+1}(D)}+|p|_{\mathcal{H}^{k+1}(D)}+h|g|_{\mathcal{H}^{k+1 / 2}(\partial D)}\right) .
\end{aligned}
$$

Here, $C_{s},\left(r_{n}, q_{n}\right), n=1, \ldots, N$ are the constants for approximation in the stochastic space inherited from Proposition 4.2 and $C_{p}$ is the constant for approximation in the physical space inherited from Lemma 4.1. The quantity $|u|_{\mathcal{H}^{k+1}(D)},|p|_{\mathcal{H}^{k+1}(D)}$ and $|g|_{\mathcal{H}^{k+1 / 2}(\partial D)}$ are the semi-norm of $u, p, g$ in the stochastic Hilbert spaces.

Proof Recall the interpolation operator $\mathcal{I}_{q}:(u, g, p) \rightarrow \mathcal{I}_{q}(u, g, p) \equiv\left(u_{q}, g_{q}, p_{q}\right)$ in the stochastic space. Denoting by $P_{h}^{s}:\left(u_{q}, g_{q}, p_{q}\right) \rightarrow P_{h}^{s}\left(u_{q}, g_{q}, p_{q}\right) \equiv\left(u_{h, q}, g_{h, q}, p_{h, q}\right)$ the point-wise projection operator for the stabilized finite element approximation in the physical space, which projects the point-wise solution $\left(u_{q}(y), g_{q}(y), p_{q}(y)\right)$ for any $y \in \Gamma$ from the Hilbert space $H^{k+1}(D) \times H^{k+1 / 2}(\partial D) \times$ $H^{k+1}(D)$ to the finite element space $X_{h}^{k} \times\left. X_{h}^{k}\right|_{\partial D} \times X_{h}^{k}$, we conclude the convergence result for the combined approximation as

$$
\begin{aligned}
& \left\|u-u_{h, q}\right\|_{\mathcal{V}(D)}+\left\|g-g_{h, q}\right\|_{\left.\mathcal{L}^{2}(\partial D)\right)}+\left\|p-p_{h, q}\right\|_{\mathcal{V}(D)} \\
& \equiv\left\|u-u_{h, q}\right\|_{L^{2}(\Gamma, V)}+\left\|g-g_{h, q}\right\|_{L^{2}\left(\Gamma ; L^{2}(\partial D)\right)}+\left\|p-p_{h, q}\right\|_{L^{2}(\Gamma, V)} \\
& =\left\|u-P_{h}^{s} I_{q} u\right\|_{L^{2}(\Gamma, V)}+\left\|g-P_{h}^{s} I_{q} g\right\|_{L^{2}\left(\Gamma ; L^{2}(\partial D)\right)}+\left\|p-P_{h}^{s} I_{q} p\right\|_{L^{2}(\Gamma, V)} \\
& \leq\left\|u-I_{q} u\right\|_{L^{2}(\Gamma, V)}+\left\|I_{q} u-P_{h}^{s} I_{q} u\right\|_{L^{2}(\Gamma, V)} \\
& \quad+\left\|g-I_{q} g\right\|_{L^{2}\left(\Gamma ; L^{2}(\partial D)\right)}+\left\|I_{q} g-P_{h}^{s} I_{q} g\right\|_{L^{2}\left(\Gamma ; L^{2}(\partial D)\right)} \\
& \quad+\left\|p-I_{q} p\right\|_{L^{2}(\Gamma, V)}+\left\|I_{q} p-P_{h}^{s} I_{q} p\right\|_{L^{2}(\Gamma, V)} \\
& =\left\|u-I_{q} u\right\|_{L^{2}(\Gamma, V)}+\left\|g-I_{q} g\right\|_{L^{2}\left(\Gamma ; L^{2}(\partial D)\right)}+\left\|p-I_{q} p\right\|_{L^{2}(\Gamma, V)} \\
& \quad+\left\|I_{q} u-P_{h}^{s} I_{q} u\right\|_{L^{2}(\Gamma, V)}+\left\|I_{q} g-P_{h}^{s} I_{q} g\right\|_{L^{2}\left(\Gamma ; L^{2}(\partial D)\right)}+\left\|I_{q} p-P_{h}^{s} I_{q} p\right\|_{L^{2}(\Gamma, V)} \\
& \leq C_{s} \sum_{n=1}^{N}\left(\frac{1}{r_{n}}\right)^{q_{n}}+C_{p}\left(\varepsilon^{1 / 2}+h^{1 / 2}\right) h^{k}\left(|u|_{\mathcal{H}^{k+1}(D)}+|p|_{\mathcal{H}^{k+1}(D)}+h|g|_{\mathcal{H}^{k+1 / 2}(\partial D)}\right),
\end{aligned}
$$

The first inequality is due to the triangular inequality, and the second one follows from using the converge results of the stabilized finite element approximation and the stochastic collocation approximation.

Using similar arguments, we have the following convergence result for the isotropic/anisotropic sparse grid stochastic collocation approximation.

Theorem 4.5 If the assumptions in Corollary 3.2. Lemma 4.1 and Proposition 4.3 are satisfied, we have the following global error estimate for stabilized finite element approximation in the physical space and isotropic/anisotropic sparse grid collocation approximation in the stochastic space

$$
\begin{aligned}
& \left\|u-u_{h, q}\right\|_{\mathcal{V}(D)}+\left\|g-\left.g_{h, q}\right|_{\left.\mathcal{L}^{2}(\partial D)\right)}+\right\| p-p_{h, q} \|_{\mathcal{V}(D)} \\
& \leq C_{s} N_{q}^{-r}+C_{p}\left(\varepsilon^{1 / 2}+h^{1 / 2}\right) h^{k}\left(|u|_{\mathcal{H}^{k+1}(D)}+|p|_{\mathcal{H}^{k+1}(D)}+h|g|_{\mathcal{H}^{k+1 / 2}(\partial D)}\right) .
\end{aligned}
$$

where $C_{p}$ is the constant for approximation in physical space inherited from Lemma 4.1, and $C_{s}, N_{q}$ and $r$ are the constants for approximation in stochastic space inherited from Proposition 4.3. 


\section{$5 \quad$ Numerical results}

In this section, we demonstrate by numerical experiments our error estimates for the approximation of the stochastic optimal Robin boundary control problem obtained in the last section. Specifically, we test the error estimates for stabilized finite element approximation in physical space and sparse grid collocation approximation in stochastic space, respectively.

The computational domain is a two dimensional unit square $x=\left(x_{1}, x_{2}\right) \in D=(0,1)^{2}$; the coefficients $a=0.01, c=1, k=1$ and the force term $f=0.1$, all constants, are fixed; the advection field $\boldsymbol{b}=\left(b_{x_{1}}, b_{x_{2}}\right)^{T}$ is a stochastic vector function, with the second component $b_{x_{2}}=0$ and the first component $b_{x_{1}}$ as a random field with finite second moment, with expectation and correlation

$$
\mathbb{E}\left[b_{x_{1}}\right](x)=x_{2}\left(1-x_{2}\right) ; \quad \mathbb{C o v}\left[b_{x_{1}}\right]\left(x, x^{\prime}\right)=\frac{x_{2}^{2}\left(1-x_{2}\right)^{2}}{10^{2}} \exp \left(-\frac{\left(x_{1}-x_{1}^{\prime}\right)^{2}}{L^{2}}\right), \quad x, x^{\prime} \in D
$$

where $L$ is the correlation length. By Karhunen-Loève expansion, $b_{x_{1}}$ can be written as

$$
\begin{aligned}
b_{x_{1}}(x, \omega)= & x_{2}\left(1-x_{2}\right)+\frac{x_{2}\left(1-x_{2}\right)}{10}\left(\frac{\sqrt{\pi} L}{2}\right)^{1 / 2} y_{1}(\omega) \\
& +\frac{x_{2}\left(1-x_{2}\right)}{10} \sum_{n=1}^{\infty} \sqrt{\lambda_{n}}\left(\sin \left(n \pi x_{1}\right) y_{2 n}(\omega)+\cos \left(n \pi x_{1}\right) y_{2 n+1}(\omega)\right),
\end{aligned}
$$

where the uncorrelated random variables $y_{n}, n \geq 1$ have zero mean and unit variance, and the eigenvalues $\lambda_{n}, n \geq 1$ decay as follows:

$$
\sqrt{\lambda_{n}}=(\sqrt{\pi} L)^{1 / 2} \exp \left(-\frac{(n \pi L)^{2}}{8}\right), \quad \forall n \geq 1
$$

As for Robin boundary condition $g$, we assume that its expectation and correlation function are given on the same segment of the boundary,

$$
\mathbb{E}[g](x)=1 ; \quad \mathbb{C o v}[g]\left(x, x^{\prime}\right)=\frac{1}{2^{2}} \exp \left(-\frac{\left(x_{1}-x_{1}^{\prime}\right)^{2}+\left(x_{2}-x_{2}^{\prime}\right)^{2}}{L^{2}}\right), \quad x, x^{\prime} \in \partial D .
$$

The Karhunen-Loève expansion of the stochastic Robin boundary condition is written, e.g. on $0 \times[0,1]$

$$
g(x, \omega)=1+\frac{1}{2}\left(\frac{\sqrt{\pi} L}{2}\right)^{1 / 2} y_{1}(\omega)+\frac{1}{2} \sum_{n=1}^{\infty} \sqrt{\lambda_{n}}\left(\sin \left(n \pi x_{2}\right) y_{2 n}(\omega)+\cos \left(n \pi x_{2}\right) y_{2 n+1}(\omega)\right),
$$

where $\lambda_{n}, n \geq 1$ are the same as in (5.3). For the sake of computation, we truncate the expansion up to $N$ terms and assume that the random variables are independent and obeying the same uniform distribution $y_{n} \sim \mathcal{U}(-\sqrt{3}, \sqrt{3}), n=1, \ldots, N$ with zero mean and unit variance.

As the first test example, let us choose the correlation length $L=1 / 4$ for both velocity $\boldsymbol{b}$ and Robin boundary condition $g$, for which we only need $n=7$ terms in both of the truncations and therefore 15 independent random variables. Using piecewise linear function space $X_{h}^{1}, h=0.025$ for stabilized finite element approximation and isotropic sparse grid collocation approximation with Clenshaw-Curtis collocation nodes as $\mathcal{S}_{q}, q=19$ in 4.23 , we can compute the solution for the stochastic advection dominated elliptic problem (2.2) on each finite element node of 2792 nodes in $D$ and each collocation node of 40001 nodes in $\Gamma$. The expectation and standard deviation of the solution can also be evaluated, as shown in Figure 5.1 .

Taking the solution as our objective function $u_{d}=u$, and solving the stabilized optimality system (4.6), we obtain the optimal solution $(u, g, p)$. The expectation and standard deviation of the stochastic Robin boundary control function $g$ is displayed on the left of Figure 5.2 , which is very close to the theoretical value $\mathbb{E}[g] \equiv \mu=1$ and $\operatorname{Var}[g] \equiv \sigma=0.4876$ computed directly from (5.5).

In order to verify the theoretical convergence rate in different finite element spaces, we choose $X_{h}^{1}$ 

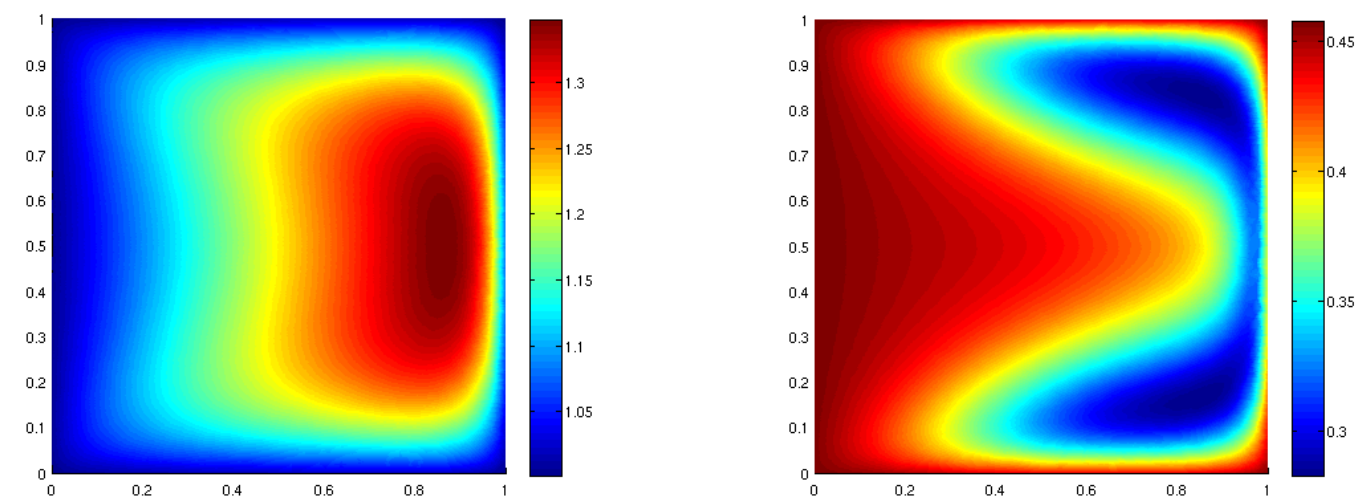

Figure 5.1: Expectation (left) and standard deviation (right) of the solution of problem (2.2)
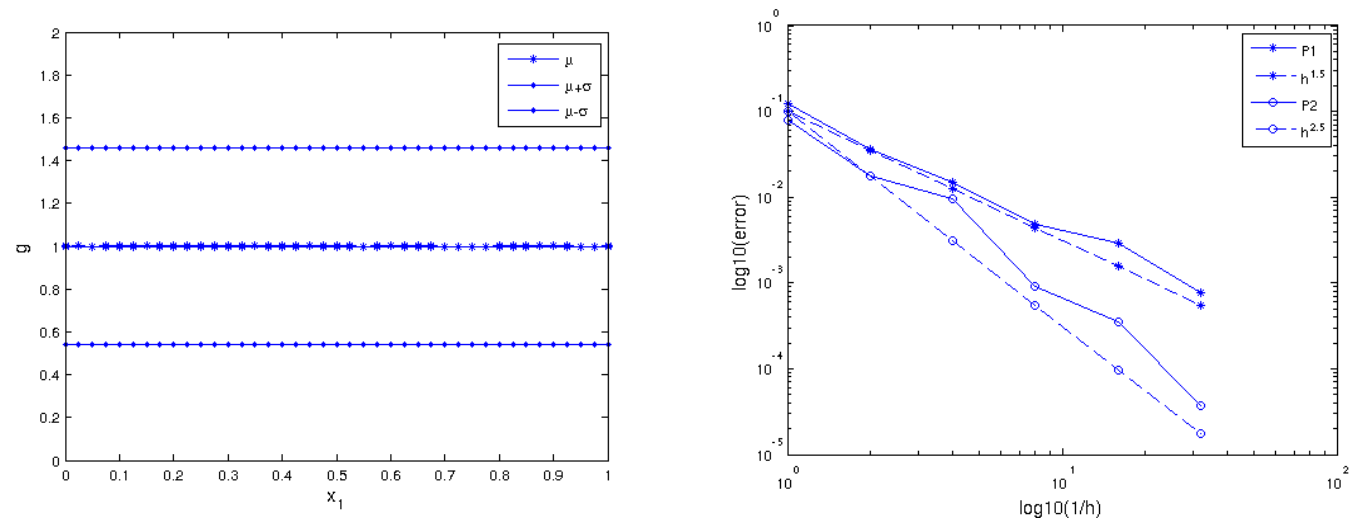

Figure 5.2: Expectation $\mu$ and standard deviation $\sigma$ of the Robin boundary condition $g$ (left), and convergence rate of the error of the solution in stabilized finite element space $X_{h}^{1}$ and $X_{h}^{2}$ (right)

and $X_{h}^{2}$, where for the second one we replace $h_{K}$ by $h_{K}^{2}$ in the specification of Péclet number $P e$ in (4.7). The series of $h$ are $h=1,1 / 2,1 / 2^{2}, 1 / 2^{3}, 1 / 2^{4}, 1 / 2^{5}$. The error is defined as

$$
\text { error }=\left\|u-u_{h, q}\right\|_{\mathcal{V}(D)}+\left\|g-g_{h, q}\right\|_{\left.\mathcal{L}^{2}(\partial D)\right)}+\left\|p-p_{h, q}\right\|_{\mathcal{V}(D)},
$$

where $u$ is computed by setting $h=1 / 2^{6}$ and $q=19, g$ is given by formula $(5.5)$, the adjoint variable $p$ is set as $0,\left(u_{h, q}, g_{h, q}, p_{h, q}\right)$ is computed by solving the optimality system (4.6). The convergence results is shown on the right of Figure 5.2, which implies that the error decays approximately with order $h^{1.5}$ for $X_{h}^{1}$ and order $h^{2.5}$ for $X_{h}^{2}$, consistently with our theoretical result in Theorem 4.5.

For simplicity, we use the same set of random variables for the expansion of $b_{x_{1}}$ and $g$ in order to test the convergence rate of the collocation approximation. The same error defined in $(5.6)$ is used. For the test of isotropic sparse grid collocation approximation, we use the series of different levels of interpolation 1, 2, 3, 4, 5, 6, 7 and set the approximated value in the deepest level as the true solution. The correlation length is set as $L=1 / 4$ and the number of random variables $\# r v=3,5,7$. The step size for the stabilized finite element approximation $h$ is set to be a relatively large value 0.25 to accelerate the computation. The error against the number of collocation nodes is displayed on the left of Figure 5.3. from which we can see that the convergence rate decreases as the number of random variables increases, and the comparison of the convergence rate with $O\left(1 / N^{2}\right)$ and $O(1 / N)$ shows that the isotropic sparse grid collocation approximation is faster than Monte Carlo method whose convergence rate is $O\left(1 / N^{1 / 2}\right)$. 

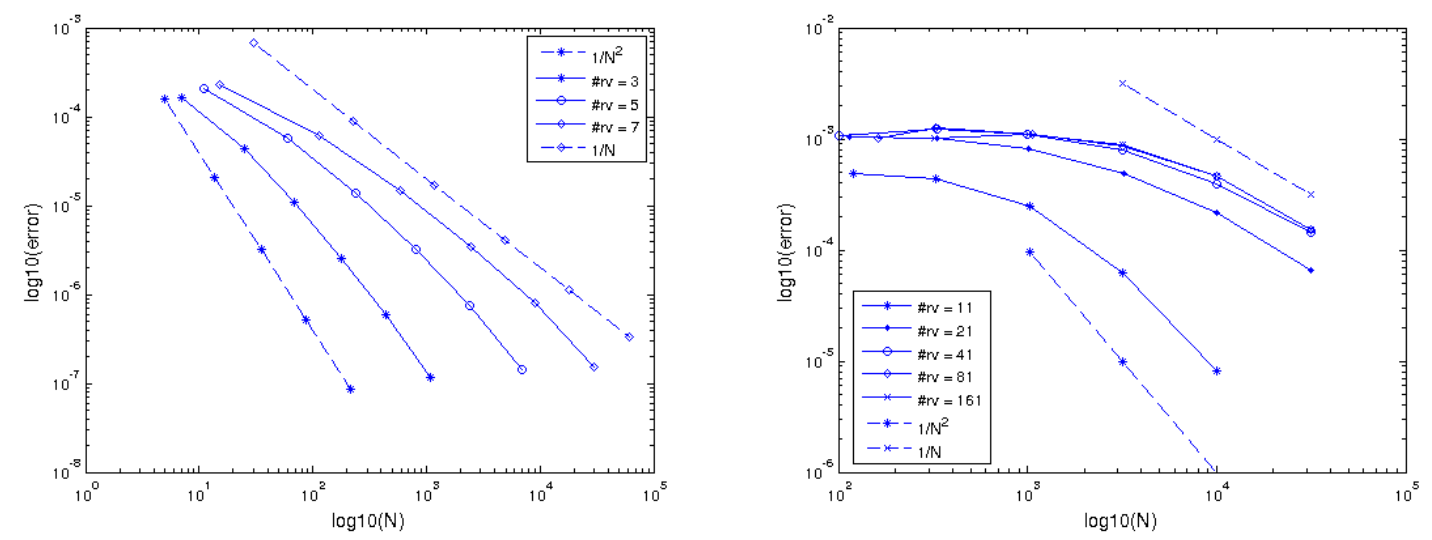

Figure 5.3: Comparison of convergence rate by isotropic sparse grid collocation approximation (left) and anisotropic sparse grid collocation approximation (right) for different stochastic dimensions

However, when the number of the random variables becomes very large, this potential advantage will fade down. In this case, we need to approximate with high interpolation level in those dimensions that are more important than the others, using the anisotropic sparse grid. On the right of Figure 5.3 , we show the convergence rate with even smaller correlation length $L=1 / 16$ for high dimensional approximation $\# r v=11,21,41,81,161$. We set a series of collocation nodes with the cardinality as $10^{2}, 10^{2.5}, 10^{3}, 10^{3.5}, 10^{4}, 10^{4.5}, 10^{5}$ and use the solution $u$ computed with $10^{5}$ collocation nodes as the true solution. The same stepsize $h=0.25$ is used. From the figure we can see that the anisotropic sparse grid breaks the "curse of dimension" in the sense of being able to taking care of very high dimensional stochastic anisotropic problems. Moreover, the convergence rate can be compared to $O\left(1 / N^{2}\right)$ for 11 dimensions and $O(1 / N)$ for over 41 dimensions, which are both higher than $O\left(1 / N^{1 / 2}\right)$. The convergence rate becomes almost the same for dimension over 41 since the randomness is captured over $99 \%$ by $n=26$ terms truncation for $L=1 / 16$, so that the left random variables play very little role.

From the above numerical results, we can conclude that the theoretical results obtained in the last section are very well verified. Meanwhile, the isotropic sparse grid stochastic collocation approximation is very efficient for stochastic optimal control problems with moderate dimensions and the application of the anisotropic sparse grid is able to deal with high dimensional stochastic problems with different weights in different dimensions (up to the order $O\left(10^{2}\right)$ ).

\section{Concluding remarks}

In this paper, we presented a stochastic optimal Robin boundary control problem constrained by an advection dominated elliptic equation. The particular uncertainties we considered arise from the background velocity of the advection term, the objective function as well as the stochastic optimal control function. We introduced the stochastic saddle point formulation and proved its equivalence to the first order necessary optimality system for the stochastic optimal control problem. The stochastic regularity with respect to the random variables was obtained thanks to Brezzi's theorem for the saddle point system. We applied stabilized finite element approximation in physical space and stochastic collocation approximation in stochastic space to discretize the optimality system. A global error estimate was obtained for the approximation. In the last part, the error estimate is verified by numerical experiments, with anisotropic sparse grid collocation approximation being highlighted for treating very high dimensional stochastic problems. Further analysis of other approximations, e.g., adaptive hierarchical stochastic collocation approximation, and applications of them to more general distributed and boundary stochastic optimal control problems, e.g., stochastic optimal control constrained by Stokes 
and Navier-Stokes equations, are ongoing.

Acknowledgement: We acknowledge the use of the Matlab packages MLife previously developed by Prof. Fausto Saleri from MOX, Politecnico di Milano and spinterp by Dr. Andreas Klimke from Universität Stuttgart http://www.ians.uni-stuttgart.de/spinterp/. This work is partially supported by FNS 200021_141034.

\section{References}

[1] R.C. Almeida and J.T. Oden. Solution verification, goal-oriented adaptive methods for stochastic advection-diffusion problems. Computer Methods in Applied Mechanics and Engineering, 199(37):2472-2486, 2010.

[2] I. Babuška, F. Nobile, and R. Tempone. A stochastic collocation method for elliptic partial differential equations with random input data. SIAM Journal on Numerical Analysis, 45(3):10051034, 2007.

[3] J. Bäck, F. Nobile, L. Tamellini, and R. Tempone. Stochastic spectral Galerkin and collocation methods for PDEs with random coefficients: A numerical comparison. Spectral and High Order Methods for Partial Differential Equations. Springer, 76:43-62, 2011.

[4] R. Becker and B. Vexler. Optimal control of the convection-diffusion equation using stabilized finite element methods. Numerische Mathematik, 106(3):349-367, 2007.

[5] P.B. Bochev and M.D. Gunzburger. Least-squares finite element methods, volume 166. Springer, 2009.

[6] M. Braack. Optimal control in fluid mechanics by finite elements with symmetric stabilization. SIAM Journal on Control and Optimization, 48:672, 2009.

[7] F. Brezzi. On the existence, uniqueness and approximation of saddle-point problems arising from lagrangian multipliers. RAIRO. Modélisation Mathématique et Analyse Numérique, 8(2):129-151, 1974.

[8] F. Brezzi and M. Fortin. Mixed and hybrid finite element methods. Springer, 1991.

[9] P. Chen, A. Quarteroni, and G. Rozza. Comparison of reduced basis method and stochastic collocation method for stochastic problems. Submitted, 2012.

[10] A. Cohen, R. DeVore, and C. Schwab. Convergence rates of best n-term galerkin approximations for a class of elliptic spdes. Found. Comput. Math, 10(6):615-646, 2010.

[11] S.S. Collis and M. Heinkenschloss. Analysis of the streamline upwind/petrov galerkin method applied to the solution of optimal control problems. CAAM TR02-01, 2002.

[12] C. Dobrzynski, T. Colin, R. Abgrall, J. Beck, F. Nobile, L. Tamellini, and R. Tempone. Implementation of optimal galerkin and collocation approximations of pdes with random coefficients. In ESAIM: Proceedings, volume 33:10-21. EDP Sciences, 2011.

[13] R. Durrett. Probability: theory and examples. Cambridge University Press, 2010.

[14] R. Glowinski and JL Lions. Exact and approximate controllability for distributed parameter systems. Cambridge University Press, 1996.

[15] M.D. Gunzburger, H.C. Lee, and J. Lee. Error estimates of stochastic optimal neumann boundary control problems. SIAM Journal on Numerical Analysis, 49:1532-1552, 2011.

[16] M. Heinkenschloss and D. Leykekhman. Local error estimates for SUPG solutions of advectiondominated elliptic linear-quadratic optimal control problems. SIAM Journal on Numerical Analysis, 47(6):4607-4638, 2010. 
[17] M. Hinze, N. Yan, and Z. Zhou. Variational discretization for optimal control governed by convection dominated diffusion equations. Journal of Computational Mathematics, 27(2-3):237$253,2009$.

[18] LS Hou, J. Lee, and H. Manouzi. Finite element approximations of stochastic optimal control problems constrained by stochastic elliptic pdes. Journal of Mathematical Analysis and Applications, 384(1):87-103, 2011.

[19] A. Klimke. Uncertainty modeling using fuzzy arithmetic and sparse grids. Universität Stuttgart. $\mathrm{PhD}$ thesis, Universität Stuttgart, 2006.

[20] A. Kunoth and C. Schwab. Analytic regularity and gpc approximation for control problems constrained by linear parametric elliptic and parabolic pdes. ETH SAM Report, 2011.

[21] J.L. Lions. Optimal control of systems governed by partial differential equations. Springer, 1971.

[22] F. Nobile, R. Tempone, and C.G. Webster. An anisotropic sparse grid stochastic collocation method for elliptic partial differential equations with random input data. SIAM Journal on Numerical Analysis, 5(46), 2008.

[23] F. Nobile, R. Tempone, and C.G. Webster. A sparse grid stochastic collocation method for partial differential equations with random input data. SIAM Journal on Numerical Analysis, 46(5):2309-2345, 2008.

[24] A. Quarteroni. Numerical models for differential problems. Springer, MS \& A, vol 2, 2009.

[25] A. Quarteroni and A. Valli. Numerical approximation of partial differential equations. Springer, 1994.

[26] Eveline Rosseel and Garth N. Wells. Optimal control with stochastic pde constraints and uncertain controls. Computer Methods in Applied Mechanics and Engineering, 213C216(0):152 - 167, 2012.

[27] C. Schwab and R. A. Todor. Karhunen-Loève approximation of random fields by generalized fast multipole methods. Journal of Computational Physics, 217(1):100-122, 2006.

[28] S.A. Smolyak. Quadrature and interpolation formulas for tensor products of certain classes of functions. In Doklady Akademii Nauk SSSR, volume 4, pages 240-243, 1963.

[29] F. Tröltzsch. Optimal control of partial differential equations: theory, methods, and applications, volume 112. American Mathematical Society, 2010.

[30] D. Xiu. Fast numerical methods for stochastic computations: a review. Communications in Computational Physics, 5(2-4):242-272, 2009.

[31] D. Xiu and J.S. Hesthaven. High-order collocation methods for differential equations with random inputs. SIAM Journal on Scientific Computing, 27(3):1118-1139, 2005. 